ALAIN BRILLARD

\title{
Asymptotic analysis of incompressible and viscous fluid flow through porous media. Brinkman's law via epi-convergence methods
}

\author{
Annales de la faculté des sciences de Toulouse $5^{e}$ série, tome $8, \mathrm{n}^{\circ} 2$ \\ (1986-1987), p. 225-252 \\ <http://www.numdam.org/item?id=AFST_1986-1987_5_8_2_225_0>
}

(C) Université Paul Sabatier, 1986-1987, tous droits réservés.

L'accès aux archives de la revue «Annales de la faculté des sciences de Toulouse » (http://picard.ups-tlse.fr/ annales/) implique l'accord avec les conditions générales d'utilisation (http://www.numdam.org/conditions). Toute utilisation commerciale ou impression systématique est constitutive d'une infraction pénale. Toute copie ou impression de ce fichier doit contenir la présente mention de copyright.

\section{Numdam}

Article numérisé dans le cadre du programme

Numérisation de documents anciens mathématiques

http://www.numdam.org/ 


\title{
Asymptotic analysis of incompressible and viscous fluid flow through porous media. Brinkman's law via epi-convergence methods.
}

\author{
ALAIN BRILlard (1)
}

Resumb. - On étudie le comportement asymptotique, lorsque $\epsilon$ tend vers 0 , d'un fluide visqueux et incompressible, s'écoulant lentement dans un milieu poreux contenant une répartition $\epsilon$-périodique d'inclusions identiques et de taille $r_{\epsilon}\left(\lim _{\epsilon \rightarrow 0}\left(r_{\epsilon} / \epsilon\right)=0\right)$. Une nouvelle justification de la loi de Brinkman est obtenue a l'aide des techniques d'épi-convergence. Les fonctions test construites apportent quelques précisions sur le comportement asymptotique de la pression interne du fluide.

ABstract. - The asymptotic behaviour, when $\epsilon$ converges to 0 , of a viscous and incompressible fluid, slowly flowing in a porous medium containing an $\epsilon$-periodic distribution of identical inclusions of size $r_{\epsilon}\left(\lim _{\epsilon \rightarrow 0}\left(r_{\epsilon} / \epsilon\right)=\right.$ 0 ), is described. An improved justification of Brinkman's law is obtained, via epi-convergence methods. Some indications concerning the behaviour of the internal pressure of the fluid are derived from the given test functions.

\section{$\S$ 1. Introduction}

The purpose of this work is to give a mathematical justification via epiconvergence methods, of Brinkman's law which describes the asymptotic behaviour of a viscous and incompressible fluid flowing through porous media with periodic structures. Let $\Omega$ be an open bounded and smooth subset of $R^{N}(N=2$ or 3$)$ and $T$ be a smooth subset of the unit ball $B(1)$ of $R^{N}$. The porous medium $\Omega_{\epsilon}$ is defined, for $\epsilon$ strictly positive by $\Omega_{\epsilon}=\Omega \backslash \bigcup_{i=1}^{I(\epsilon)} \bar{T}_{\epsilon i}$, where $\bigcup_{i} T_{\epsilon i}$ is a cloud of $\epsilon$-periodically distributed identical $r_{\epsilon}$-homothetics of $T\left(0<r_{\epsilon}<\epsilon / 2\right)$

(1) Faculté des Sciences et Techniques, 4 rue des Frères Lumière - 68093 Mulhouse Cédex 
A. Brillard

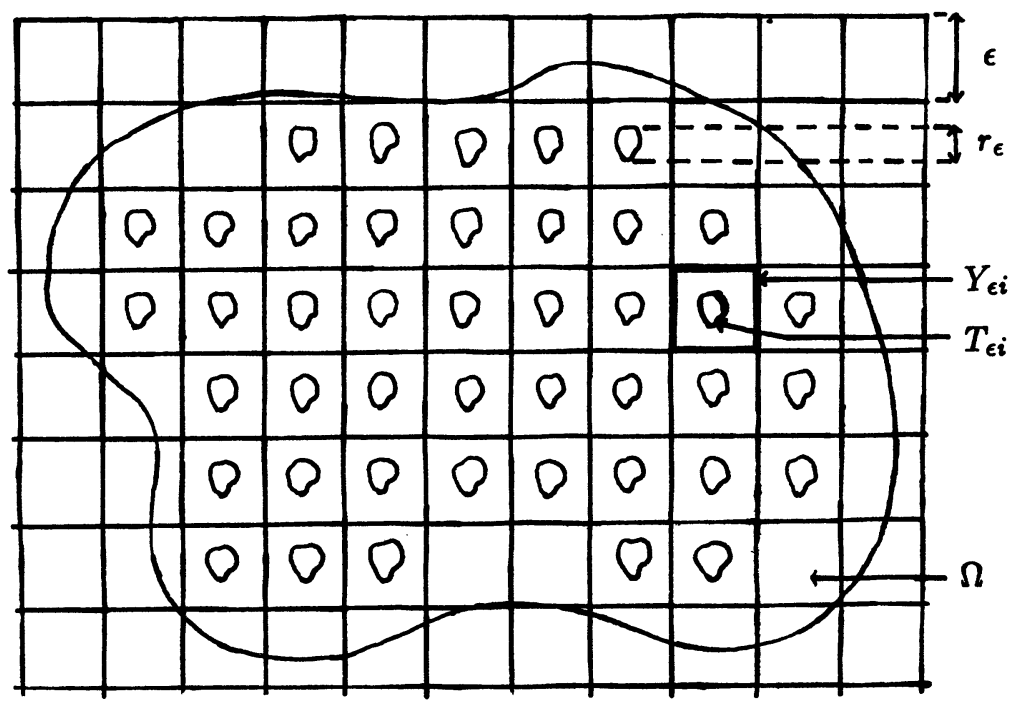

The total number $I(\epsilon)$ of inclusions is equivalent to $\operatorname{Vol}(\Omega) / \epsilon^{N}$.

\section{A) Fluid flow through porous media}

An incompressible and viscous fluid flows slowly in the porous medium $\Omega_{\epsilon}$ under the action of external forces $\vec{f}\left(\vec{f}\right.$ belongs to $\left.\left(L^{2}(\Omega)\right)^{N}\right)$. Moreover, the fluid is supposed to stick to the fixed boundary $\partial \Omega$ and to the boundary of the inclusions $\partial T_{\epsilon i}$. Therefore, the velocity $\vec{u}_{\epsilon}$ of the fluid is the solution of the Stokes equation in $\Omega_{\epsilon}$, with Dirichlet boundary conditions on $\partial \Omega_{\epsilon}$ (for simplicity, the viscosity coefficient has been normalized to 1 )

$$
\begin{cases}-\Delta \vec{u}_{\epsilon}=-\operatorname{grad} p_{\epsilon}+\vec{f} & \text { in } \Omega_{\epsilon}\left(p_{\epsilon}\right. \text { is the internal pressure) } \\ \operatorname{div} \vec{u}_{\epsilon}=0 & \text { in } \Omega_{\epsilon} \\ \vec{u}_{\epsilon}=\overrightarrow{0} & \text { on } \partial \Omega_{\epsilon}=\partial \Omega \cup\left(\bigcup_{i} \partial T_{\epsilon i}\right) .\end{cases}
$$

In [10], Marchenko and Hrouslov proved the following result concerning the behaviour of $\vec{u}_{\epsilon}$, when the parameter $\epsilon$ goes to 0 (see also $[8,9]$ ).

THEOREM 1.1. - Suppose $\lim _{\epsilon \rightarrow 0} \frac{r_{\epsilon}}{\epsilon}=0$. Then the sequence $\left(P^{\epsilon} \vec{u}_{\epsilon}\right)_{\epsilon}$ of canonical extensions of $\vec{u}_{\epsilon}$ (taking the value $\overrightarrow{0}$ on the inclusions), converges in the weak topology of $\left(H_{o}^{1}(\Omega)\right)^{N}$ to the solution $\vec{u}_{o}$ of Brinkman's law

$$
\mid \begin{array}{ll}
-\Delta \vec{u}_{o}+M \vec{u}_{o}=-\operatorname{grad} p_{o}+\vec{f} & \text { in } \Omega, \\
\operatorname{div} \vec{u}_{o}=0 & \text { in } \Omega, \\
\vec{u}_{o} \in\left(H_{o}^{1}(\Omega)\right)^{N} . &
\end{array}
$$


where $M$ is the positive symmetric matrix defined by : for every $k, \ell$ in $\{1, \ldots, N\}$

$$
M_{k \ell}=\lim _{\epsilon \rightarrow 0}\left(\frac{1}{\epsilon^{N}} \int_{B(\epsilon / 4) \backslash T_{\epsilon}} \operatorname{grad} \vec{w}_{\epsilon}^{k} \cdot \operatorname{grad} \vec{w}_{\epsilon}^{\ell} d x\right),
$$

$\vec{w}_{\epsilon}^{k}$ being the solution of the local problem

$$
\mid \begin{array}{ll}
-\Delta \vec{w}_{\epsilon}^{k}=\operatorname{grad} q_{\epsilon}^{k} & \text { in } B(\epsilon / 4) \backslash T_{\epsilon}, \\
\operatorname{div} \vec{w}_{\epsilon}^{k}=0 & \text { in } B(\epsilon / 4) \backslash T_{\epsilon}, \\
\vec{w}_{\epsilon}^{k}=\overrightarrow{0} \text { on } \partial T_{\epsilon}, & \\
\vec{w}_{\epsilon}^{k}=\vec{e}_{k} \text { on } \partial B(\epsilon / 4) & \left(\vec{e}_{k} \text { is the } k^{\text {th }} \text { canonical vector of } R^{N}\right) .
\end{array}
$$

Marchenko and Hrouslov's proof consists essentially to build, for every smooth divergence-free function $\vec{v}$ in $\left(H_{o}^{1}(\Omega)\right)^{N}$, a divergence-free function $\vec{v}_{\epsilon}^{o}$ in $\left(H_{o}^{1}\left(\Omega_{\epsilon}\right)\right)^{N}$, such that

i) the sequence $\left(P^{\epsilon} \vec{v}_{\epsilon}^{o}\right)_{\epsilon}$ of canonical extensions of $\vec{v}_{\epsilon}^{o}$, taking the value $\overrightarrow{0}$ on the inclusions, converges to $\vec{v}$ in the weak topology of $\left(H_{o}^{1}(\Omega)\right)^{N}$,

ii) $\lim _{\epsilon \rightarrow 0}\left(\int_{\Omega_{\epsilon}} \operatorname{grad} \vec{u}_{\epsilon} \cdot \operatorname{grad} \vec{v}_{\epsilon}^{\circ} d x\right)=\int_{\Omega} \operatorname{grad} \vec{u}_{o} \cdot \operatorname{grad} \vec{v} d x+$

$$
+\sum_{k \ell} \int_{\Omega} M_{k \ell}\left(\vec{u}_{o}\right)_{k} v_{\ell} d x
$$

and then to use the density of smooth divergence-free functions in the closed subspace $V(\Omega)$ of $\left(H_{o}^{1}(\Omega)\right)^{N}$ containing the divergence-free functions. (See Remark 2.11. later on).

The purpose of the first part of this work is to prove this Theorem 1.1. by means of epi-convergence theory. Let us now present this variational convergence.

\section{B) Epi-convergence}

DEFINITION $1.2[2],[6]$. - Let $(X, \tau)$ be a metric vector space and $\left(F^{\epsilon}\right)_{\epsilon}$ a sequence of functionals from $X$ into $\bar{R}$. Then, $\left(F^{\epsilon}\right)_{\epsilon}$ epi $i_{r}$-converges to a functional $F$ from $X$ into $\bar{R}$ if

$$
\forall x \in X:\left(\tau-\varliminf_{\epsilon \rightarrow 0} F^{\epsilon}\right)(x)=\left(\tau-\varlimsup_{\epsilon \rightarrow 0} F^{\epsilon}\right)(x)=F(x),
$$




\section{A. Brillard}

where

$$
\begin{aligned}
& \left(\tau-\varliminf_{\epsilon \rightarrow 0} F^{\epsilon}\right)(x)=\min _{x_{\epsilon} \underset{\epsilon \rightarrow 0}{\epsilon \rightarrow \infty}} \varliminf_{\epsilon \rightarrow 0} F^{\epsilon}\left(x_{\epsilon}\right), \\
& \left(\tau-\varlimsup_{\epsilon \rightarrow 0} F^{\epsilon}\right)(x)=\min _{x_{\epsilon}{\underset{\tau}{\epsilon \rightarrow 0}}_{\epsilon \rightarrow 0}} \varlimsup_{\epsilon \rightarrow 0} F^{\epsilon}\left(x_{\epsilon}\right) .
\end{aligned}
$$

This equality (5) is satisfied if and only if the two assertions are fullfilled

$$
\begin{aligned}
& \forall x \in X, \exists x_{\epsilon}^{\circ} \underset{\epsilon \rightarrow 0}{\stackrel{\tau}{\longrightarrow}} x: \varlimsup_{\epsilon \rightarrow 0} F^{\epsilon}\left(x_{\epsilon}^{\circ}\right) \leq F(x), \\
& \forall x \in X, \forall x_{\epsilon} \underset{\epsilon \rightarrow 0}{\stackrel{\tau}{\longrightarrow}} x: \varliminf_{\epsilon \rightarrow 0} F^{\epsilon}\left(x_{\epsilon}\right) \geq F(x) .
\end{aligned}
$$

This variational convergence is well-fitted to the asymptotic analysis of minimization problems, since we have the following result.

THEOREM 1.3. - ([2] Theorem 1.10). Suppose $\left(F^{\epsilon}\right)_{\epsilon}$ epi $i_{\tau}$-converges to $F$. Let $\left(o_{\epsilon}\right)_{\epsilon}$ be a sequence converging to 0 and for every $\epsilon, \bar{x}_{\epsilon}$ a $o_{\epsilon}$-minimizer of $F^{\epsilon}$, that is

$$
F^{\epsilon}\left(\bar{x}_{\epsilon}\right) \leq \inf _{X} F^{\epsilon}(x)+o_{\epsilon}
$$

Then, for every $\tau$-converging subsequence $\left(\bar{x}_{\epsilon^{\prime}}\right)_{\epsilon^{\prime}}$ of $\left(\bar{x}_{\epsilon}\right)$, with $\bar{x}=\lim _{\epsilon^{\prime} \rightarrow 0} \bar{x}_{\epsilon^{\prime}}$ :

$$
F(\bar{x})=\min _{x \in X} F(x)
$$

and moreover

$$
\lim _{\epsilon^{\prime} \rightarrow 0} F^{\epsilon^{\prime}}\left(\bar{x}_{\epsilon^{\prime}}\right)=F(\bar{x})
$$

Notice that the topology $\tau$ is not a priori imposed but must be choosen so that the sequence $\left(x_{\epsilon}\right)_{\epsilon}$ is $\tau$-relatively compact.

The next result shows the stability of epi-convergence with respect to continuous perturbations.

PROPOSITION 1.4.- ([2] Theorem 2.15). Suppose $\left(F^{\epsilon}\right)_{\epsilon}$ epi-converges to $F$ and $G$ is $\tau$-continuous on $X$. Then $\left(F^{\epsilon}+G\right)_{\epsilon}$ epi $i_{\tau}$-converges to $F+G$.

Our approach improves on Marchenko and Hrouslov's proof in two directions :

i) The test function $\vec{v}_{\epsilon}^{o}$ appearing in (6) is a little simpler than their test function, when $T$ is equal to $B(1)$. This is a consequence of the explicit 
computation of the solution $\left(\vec{w}_{\epsilon}^{k}, q_{\epsilon}^{k}\right)$ of the local problem (4) given in the Appendix.

ii) The use of this theoretical framework, that is epi-convergence, which is related to the $G$-convergence of the linked operators (see $[2,6]$ ), simultaneously furnishes :

1) the behaviour of the kinetic energy of the fluid,

2) the convergence of the solution of the Stokes evolution problem in porous media,

3) the convergence of the spectra of the linked operators,

4) some indications about first-order correctors. (see [4] for these results).

\section{C) Convergence of the internal pressure}

Theorem 1.1 asserts the convergence of a trivial extension $P^{\epsilon} \vec{u}_{\epsilon}$ of the solution $\vec{u}_{\epsilon}$ of $(1)$ in the fixed space $\left(H_{o}^{1}(\Omega)\right)^{N}$, equiped with its weak topology. Then, the problem, which immediately raises is : does there exists an extension of the dual variable grad $p_{\epsilon},\left(\operatorname{grad} p_{\epsilon}\right.$ belongs to $\left.\left(H^{-1}\left(\Omega_{\epsilon}\right)\right)^{N}\right)$ which converges to grad $p_{o}$ in the fixed space $\left(H^{-1}(\Omega)\right)^{N}$ ?

In [13], Tartar solved this problem in the case : $r_{\epsilon}=\lambda \epsilon(0<\lambda<1 / 2)$. His proof is based on the explicit construction of a linear, continuous, restriction operator $R^{\epsilon}$ from $\left(H_{o}^{1}(\Omega)\right)^{N}$ into $\left(H_{o}^{1}\left(\Omega_{\epsilon}\right)\right)^{N}$ such that, for every $\vec{v}$ in $\left(H_{o}^{1}(\Omega)\right)^{N}$

$$
\lim _{\epsilon \rightarrow 0}<\operatorname{grad} p_{\epsilon}, R^{\epsilon} \vec{v}>=<\operatorname{grad} p_{o}, \vec{v}>
$$

Then $R^{\epsilon *}\left(\operatorname{grad} p_{\epsilon}\right)$, which is still a gradient (see theorem 3.1. later on), converges in the weak (and in fact strong) topology of $\left(H^{-1}(\Omega)\right)^{N}$.

In the present case, that is when $\lim _{\epsilon \rightarrow 0}\left(r_{\epsilon} / \epsilon\right)=0$, we did not succeed in such a construction : nevertheless, we shall present in part III of this study, two operators $R_{j}^{\epsilon}(j=1,3)$

$$
\begin{aligned}
& R_{1}^{\epsilon}: X_{1}=\left(H_{o}^{1}(\Omega)\right)^{N} \cap\left(C^{1}(\bar{\Omega})\right)^{N} \rightarrow\left(H_{o}^{1}\left(\Omega_{\epsilon}\right)\right)^{N}, \\
& R_{3}^{\epsilon}: X_{3}=\left(H_{o}^{1}(\Omega)\right)^{N} \cap\left(C^{\circ}(\bar{\Omega})\right)^{N} \rightarrow\left(H_{o}^{1}\left(\Omega_{\epsilon}\right)\right)^{N},
\end{aligned}
$$

such that for every $\vec{v}$ in $X_{j}(j=1,3)$ :

$$
\lim _{\epsilon \rightarrow 0}<\operatorname{grad} p_{\epsilon}, R_{j}^{\epsilon} \vec{v}>=<\operatorname{grad} p_{o}, \vec{v}>.
$$




\section{A. Brillard}

Hence, the extension $R_{j}^{\epsilon^{*}}$ (grad $p_{\epsilon}$ ) (which is not yet a gradient) converges to $\operatorname{grad} p_{o}$ in the fixed space $X_{j^{\prime}}\left(X_{j^{\prime}}\right.$ is the dual space of $\left.X_{j}\right)$.

The proofs crucially require the smoothness of $\vec{v}$ in $X_{j}$.

\section{D) Notations}

The following spaces or notations are used throughout this study

$C^{\circ}(\bar{\Omega})$ is the space of continuous functions in $\bar{\Omega}$,

$C^{1}(\bar{\Omega})$ is the subspace of $C^{\circ}(\bar{\Omega})$ consisting of all functions whose first partial derivatives are bounded and uniformly continuous in $\bar{\Omega}[1]$,

$C_{o}^{\infty}(\Omega)$ is the space of smooth functions in $\Omega$. These functions have continous partial derivatives of any order in $\Omega$ and a compact support in $\Omega$,

$\left(L^{2}(\Omega)\right)^{N}$ is the space of Lebesgue measurable vector-valued functions whose components are square-integrable in $\Omega$,

$\left(H^{1}(\Omega)\right)^{N}$ is the Sobolev space of Lebesgue measurable vector-valued functions in $\left(L^{2}(\Omega)\right)^{N}$, with derivatives (in the distributional sense) in $L^{2}(\Omega)[1]$,

$W^{1,1}(\Omega)$ is the Sobolev space of functions in $L^{1}(\Omega)$ with derivatives in $L^{1}(\Omega)[1]$, its dual space is denoted by $W^{-1, \infty}(\Omega)$,

$\mathcal{V}(\Omega)$ is the set of smooth, divergence-free vector-valued functions in $\Omega$ :

$$
\mathcal{V}(\Omega)=\left\{\vec{u} \in\left(C_{0}^{\infty}(\Omega)\right)^{N} / \operatorname{div} \vec{u}=0 \text { in } \Omega\right\}
$$

$V(\Omega)$ is the completion of $\nu(\Omega)$ with respect to the strong topology of

$$
\left(H_{o}^{1}(\Omega)\right)^{N}: V(\Omega)=\left\{\vec{u} \in\left(H_{o}^{1}(\Omega)\right)^{N} / \operatorname{div} \vec{u}=0 \text { in } \Omega\right\}[7],[14] ;
$$

$Y$ is the unit cube of $R^{N}:\left[-\frac{1}{2}, \frac{1}{2}\right]^{N}$, while $Y_{\epsilon}$ is its $\epsilon$-homothetic.

The characteristic function of a set $A$ is denoted by $\chi_{A}$ :

$$
\chi_{A}(x)=\mid \begin{aligned}
& 1 \text { if } x \text { belongs to } A, \\
& 0 \text { otherwise. }
\end{aligned}
$$

The indicator function of a set $A$ is a denoted by $I_{A}$ :

$$
I_{A}(x)=\mid \begin{aligned}
& 0 \text { if } x \text { belongs to } A, \\
& +\infty \text { otherwise. }
\end{aligned}
$$




\section{Asymptotic analysis of incompressible}

Let $H$ be a convex function defined on a locally convex space $V$ (with dual space $V^{\prime}$ ), the subdifferential operator $\partial H$ is defined by

$$
\partial H(u)=\left\{u^{\prime} \in V^{\prime} / \forall v \in V: H(v) \geq H(u)+\left\langle u^{\prime}, v-u\right\rangle\right\} .
$$

Let me finally express my thanks to $\mathrm{H}$. Attouch for his constant encouragement and more specially for the duality approach in part III, and to F. Murat for stimulating discussions.

\section{$\S$ II. Brinkman's law}

We suppose here that the common size $r_{\epsilon}$ of the identical inclusions $T_{\epsilon i}$ satisfies

$$
\lim _{\epsilon \rightarrow 0} \frac{r_{\epsilon}}{\epsilon}=0 .
$$

If $P^{\epsilon}$ is the canonical imbedding from $\left(H_{o}^{1}\left(\Omega_{\epsilon}\right)\right)^{N}$ into $\left(H_{o}^{1}(\Omega)\right)^{N}$ :

$$
P^{\epsilon} \vec{u}=\mid \begin{aligned}
& \vec{u} \text { in } \Omega_{\epsilon}, \\
& \overrightarrow{0} \text { in } \bigcup_{i} T_{\epsilon i},
\end{aligned}
$$

the extension $P^{\epsilon} \vec{u}_{\epsilon}$ of $\vec{u}_{\epsilon}$, solution of (1), satisfies

LEMMA 2.1. - a) $P^{\epsilon} \vec{u}_{\epsilon}$ is the solution of :

$$
\min _{\left(H_{o}^{1}(\Omega)\right)^{N}}\left(F^{\epsilon}(\vec{u})-\int_{\Omega} \vec{f} \cdot \vec{u} d x\right),
$$

where $F^{\epsilon}$ is defined on $\left(H_{o}^{1}(\Omega)\right)^{N}$ by

$$
F^{\epsilon}(\vec{u})=\frac{1}{2} \int_{\Omega}|\operatorname{grad} \vec{u}|^{2} d x+I_{V\left(\Omega_{e}\right)}(\vec{u})
$$

b) The sequence $\left(P^{\epsilon} \vec{u}_{\epsilon}\right)_{\epsilon>0}$ is bounded in $\left(H_{o}^{1}(\Omega)\right)^{N}$ and relatively compact in the weak topology of $\left(H_{o}^{1}(\Omega)\right)^{N}$.

Proof of Lemma 2.1. - a) is an immediate consequence of (1).

b) From a) we infer

$$
\int_{\Omega}\left|\operatorname{grad} P^{\epsilon} \vec{u}_{\epsilon}\right|^{2} d x \leq \int_{\Omega} \vec{f} . P^{\epsilon} \vec{u}_{\epsilon} d x
$$




\section{A. Brillard}

Poincaré's inequality [1] implies the existence of a constant $C(\vec{f}, \Omega)$ such that

$$
\left\|P^{\epsilon} \vec{u}_{\epsilon}\right\|_{\left(H_{o}^{1}(\Omega)\right)^{N}} \leq C(\vec{f}, \Omega) .
$$

Our main result is

THEOREM 2.2.- a) The sequence $\left(F^{\epsilon}\right)_{\epsilon}$ defined by (9) epi $i_{w-\left(H_{o}^{1}(\Omega)\right)^{N-}}$ converges to the functional $F$ defined on $\left(H_{o}^{1}(\Omega)\right)^{N}$ by

$$
F(\vec{u})=\frac{1}{2} \int_{\Omega}|\operatorname{grad} \vec{u}|^{2} d x+\frac{1}{2} \sum_{k \ell} \int_{\Omega} M_{k \ell} u_{k} u_{\ell} d x+I_{V(\Omega)}(\vec{u}),
$$

where $M$ is the matrix given by (3).

b) Let $\vec{f}$ be any element of $\left(L^{2}(\Omega)\right)^{N}$, then the sequence $\left(P^{\epsilon} \vec{u}_{\epsilon}\right)_{\epsilon}$, where $P^{\epsilon} \vec{u}_{\epsilon}$ is the canonical extension of the solution $\vec{u}_{\epsilon}$ of (1), converges in the weak topology of $\left(H_{o}^{1}(\Omega)\right)^{N}$ to the solution $\vec{u}_{o}$ of the minimization problem

$$
\min _{\left(H_{o}^{1}(\Omega)\right)^{N}}\left(F(\vec{u})-\int_{\Omega} \vec{f} . \vec{u} d x\right),
$$

that is, the solution $\vec{u}_{o}$ of Brinkman's law (2).

Moreover, the sequence $\left(\int_{\Omega_{\epsilon}}\left|\operatorname{grad} \vec{u}_{\epsilon}\right|^{2} d x\right)_{\epsilon}$ of kinetic energies of the fluid, converges to $\int_{\Omega}\left|\operatorname{grad} \vec{u}_{o}\right|^{2} d x+\sum_{k \ell} \int_{\Omega} M_{k \ell}\left(\vec{u}_{o}\right)_{k}\left(\vec{u}_{o}\right)_{\ell} d x$.

Let us precise the limit problem (2).

Remark 2.3. - Trivially the matrix $M$, given in (3), depends on the size $r_{\epsilon}$ and the shape of the inclusions. Indeed, there exists a critical size $r_{\epsilon}^{c}$ of the inclusions

$$
r_{\epsilon}^{c}=\mid \begin{array}{ll}
C \epsilon^{3} & \text { if } N=3, \\
\exp \left(-C \epsilon^{-2}\right) & \text { if } N=2,\left(C \in R^{+*}\right),
\end{array}
$$

such that

1) if $\lim _{\epsilon \rightarrow 0}\left(r_{\epsilon} / r_{\epsilon}^{c}\right)=0$, the matrix $M$ is null and $\vec{u}_{o}$ is the solution of the Stokes problem in $\Omega$. The inclusions are too small to perturb the fluid flow;

2) if $\lim _{\epsilon \rightarrow 0}\left(r_{\epsilon} / r_{\epsilon}^{c}\right)=+\infty, \vec{u}_{o}$ is equal to $\overrightarrow{0}$. In this case, $\left(P^{\epsilon} \vec{u}_{\epsilon}\right)_{\epsilon}$ converges to $\overrightarrow{0}$ in the strong topology of $\left(H_{o}^{1}(\Omega)\right)^{N}$. Notice that the present condition on $r_{\epsilon}$ is fulfilled when $r_{\epsilon}$ is equivalent to $\epsilon$ (for a detailed study of this important case, which leads to Darcy's law, we refer to $[4],[8],[9],[12])$; 
3) if $\lim _{\epsilon \rightarrow 0}\left(r_{\epsilon} / r_{\epsilon}^{c}\right)$ belongs to $R^{+*}$, the limit law (2) contains a "strange term", in the terminology of [5], which takes the more precise values :

- if $N=3$, for every $k$ and $\ell$ in $\{1, \ldots, N\}$

$$
M_{k \ell}=\alpha_{3} \int_{R^{3}} \operatorname{grad} \vec{w}^{k} \cdot \operatorname{grad} \vec{w}^{\ell} d x,
$$

where $\vec{w}^{k}$ is the solution of

$$
\min _{\substack{\vec{w} \in\left(H_{0}^{1}\left(R^{3}\right)\right)^{3}, \operatorname{div} \vec{w}=0 \text { in } R^{3}, \vec{w}=\vec{e}_{k} \text { on } T,}} \int_{R^{3} \backslash T}|\operatorname{grad} \vec{w}|^{2} d x,
$$

- if $N=2$ and if $r_{\epsilon}=\lambda \exp \left(-\frac{\mu}{\epsilon^{2}}\right)$, there exists a constant $C_{k}$ depending only on $\mu$ such that $M_{k k}=C_{k}$ (see the computation of the local solution $\vec{w}_{\epsilon}^{k}$ in the Appendix and Lemma 2.4. of [3]).

Proof of Theorem 2.2. - Assertion b) is a consequence of assertion a) thanks to :

i) the continuity, for the weak topology of $\left(H_{o}^{1}(\Omega)\right)^{N}$, at least, of the "perturbation"

$$
\vec{u} \rightarrow \int_{\Omega} \vec{f} \cdot \vec{u} d x \text { (see Proposition 1.4.) }
$$

ii) the strict convexity and the coerciveness of the functional $F$ (which imply that :

- the solution $\vec{u}_{o}$ of the limit minimization problem (2) is unique,

- the whole sequence $\left(P^{\epsilon} \vec{u}_{\epsilon}\right)_{\epsilon}$ converges to $\vec{u}_{o}$, in the weak topology of $\left.\left(H_{o}^{1}(\Omega)\right)^{N}\right)$,

iii) the properties of the epi-convergence recalled in Theorem 1.3.

For the proof of assertion a), notice first that $V(\Omega)$ is a closed subspace of $\left(H_{o}^{1}(\Omega)\right)^{N}$, for the weak topology of $\left(H_{o}^{1}(\Omega)\right)^{N}$. Then for every $\vec{v}$ in $\left(H_{o}^{1}(\Omega)\right)^{N}$, which is not divergence-free in $\Omega$, one derives

$$
\left(\tau-\varlimsup_{\epsilon \rightarrow 0} F^{\epsilon}\right)(\vec{v})=\left(\tau-\varlimsup_{\epsilon \rightarrow 0} F^{\epsilon}\right)(\vec{v})=+\infty
$$

where $\tau$ denotes the weak topology of $\left(H_{o}^{1}(\Omega)\right)^{N}$. 


\section{A. Brillard}

Hence, one has only to deal with divergence-free functions and more precisely to prove the special form of assertions (6) and (7)

$$
\begin{aligned}
& \forall \vec{v} \in V(\Omega), \exists P^{\epsilon} \vec{v}_{\epsilon}^{o} \frac{w-\left(H_{o}^{1}(\Omega)\right)^{N}}{\epsilon \rightarrow 0} \vec{v}, \vec{v}_{\epsilon}^{o} \in V\left(\Omega_{\epsilon}\right): \varlimsup_{\epsilon \rightarrow 0} F^{\epsilon}\left(\vec{v}_{\epsilon}^{o}\right) \leq F(\vec{v}), \\
& \forall \vec{v} \in V(\Omega), \forall P^{\epsilon} \vec{v}_{\epsilon} \frac{w-\left(H_{o}^{1}(\Omega)\right)^{N}}{\epsilon \rightarrow 0} \vec{v}, \vec{v}_{\epsilon} \in V\left(\Omega_{\epsilon}\right): \varliminf_{\epsilon \rightarrow 0} F^{\epsilon}\left(\vec{v}_{\epsilon}\right) \geq F(\vec{v}) .
\end{aligned}
$$

Before introducing the appropriate test function $\vec{v}_{\epsilon}^{b}$ satisfying (11), we need the following property of divergence-free functions

THEOREM 2.4. - See Theorem 4.9. of [10] p. 236 (see also [14]). Let $\Omega$ be any bounded open and smooth subset of $R^{3}$ and $\vec{w}$ be any divergencefree function in $\left(L^{2}(\Omega)\right)^{3}$. Then, there exists a divergence-free function $\widehat{\vec{w}}$ in $\left(H^{1}(\Omega)\right)^{3}$ such that

$$
\begin{aligned}
& \widehat{\vec{w}} \cdot \vec{n}=0 \text { on } \partial \Omega,(\vec{n} \text { is the outer normal to the boundary } \partial \Omega) \\
& \text { curl } \widehat{\vec{w}}=\vec{w} \text { in } \Omega, \\
& \|\widehat{\vec{w}}\|_{\left(H^{1}(\Omega)\right)^{3}} \leq C(\Omega)\|\vec{w}\|_{\left(L^{2}(\Omega)\right)^{3}},
\end{aligned}
$$

where $C(\Omega)$ is a constant independant of $\vec{w}$ in $\left(L^{2}(\Omega)\right)^{3}$.

When $\Omega$ is a ball $B(r)$, we have the more precise estimates on the divergence-free function $\widehat{\vec{w}}$ defined in the Theorem 2.4 .

Proposition 2.5. - If $N=3:$ for every divergence-free function $\vec{w}$ in $\left(L^{2}(B(r))\right)^{3}$, there exists a divergence-free function $\vec{w}$ in $\left(H^{1}(B(r))\right)^{3}$ such that

$$
\begin{aligned}
& \widehat{\widehat{\vec{w}}} \cdot \vec{n}=0 \text { on } \partial B(r), \\
& \text { curl } \widehat{\vec{w}}=\vec{w} \text { in } B(r), \\
& \|\widehat{\vec{w}}\|_{\left.L^{2}(B(r))\right)^{3}} \leq C . r\|\vec{w}\|_{\left(L^{2}(B(r))\right)^{3}}, \\
& \|\operatorname{grad} \widehat{\vec{w}}\|_{\left(L^{2}(B(r))\right)^{\circ} \leq C\|\vec{w}\|_{\left.L^{2}(B(r))\right)^{3}}}
\end{aligned}
$$

where $C$ is a constant independant of $r$ and $\vec{w}$.

If $N=2$ for every divergence-free function $\vec{w}$ in $\left(L^{2}(B(r))\right)^{2}$, there exists a function $\widehat{w}$ in $H^{1}(B(r))$ such that

$$
\begin{aligned}
& \widehat{w}=0 \text { on } \partial B(r), \\
& \text { curl } \widehat{w}=\vec{w} \text { in } B(r), \\
& \|\widehat{w}\|_{L^{2}(B(r))} \leq C . r\|\vec{w}\|_{\left(L^{2}(B(r))\right)^{2}} \\
& \|\operatorname{grad} \widehat{w}\|_{\left(L^{2}(B(r))\right)^{2}} \leq C\|\vec{w}\|_{\left(L^{2}(B(r))\right)^{2}} \\
& -234-
\end{aligned}
$$


Proof of Proposition 2.5. - Suppose $N=3$. Consider : $\vec{w}_{r}(y)=\vec{w}(r y) \frac{1}{r}$ for $y$ in $B(1) . \vec{w}_{r}$ is divergence-free in $B(1)$ and belongs to $\left(L^{2}(B(1))\right)^{3}$. From theorem 2.4., there exists a function $\widehat{\vec{w}}_{r}$ such that

$$
\begin{aligned}
& \widehat{\vec{w}}_{r} \cdot \vec{n}=0 \text { on } \partial B(1) \\
& \text { curl } \widehat{\vec{w}}_{r}=\vec{w}_{r} \text { in } B(1) \\
& \left\|\widehat{\vec{w}}_{r}\right\|_{\left(H^{1}(B(1))\right)^{3}} \leq C(B(1))\left\|\vec{w}_{r}\right\|_{\left(L^{2}(B(1))\right)^{3}}
\end{aligned}
$$

Then take $\widehat{\vec{w}}$ defined by : $\widehat{\vec{w}}(x)=\widehat{\vec{w}}_{r}\left(\frac{x}{r}\right) r$. Suppose $N=2$. Consider the function $(\vec{w}, 0)$ and apply the preceding argument.

The proof of the assertion a) of the Theorem 2.2. will be divided in three steps :

$1^{\text {rst }}$ step - Verification of (11) when $\alpha_{N}$ is finite, with

$$
\alpha_{N}=\mid \begin{aligned}
& \lim _{\epsilon \rightarrow 0} r_{\epsilon} / \epsilon^{3} \text { if } N=3, \\
& \lim _{\epsilon \rightarrow 0} 1 /\left(\epsilon^{2}\left|\log r_{\epsilon}\right|\right) \text { if } N=2,
\end{aligned}
$$

$2^{\text {nd }}$ step - Verification of (12) when $\alpha_{N}$ is finite.

$3^{\text {rd }}$ step $-\alpha_{N}$ is equal to $+\infty$.

$1^{\text {rst }}$ step

Let us first suppose that $\vec{v}$ is in $\nu(\Omega)$ and denote

$$
\vec{v}_{\epsilon}^{o}(x)=\mid \begin{aligned}
& \vec{v}(x)-\sum_{k}\left(\vec{e}_{k}-\vec{w}_{\epsilon}^{k}(x)\right) v_{k}\left(x_{\epsilon i}\right)-\operatorname{curl}\left(\phi_{\epsilon i} \widehat{\vec{v}}_{\epsilon i}\right)(x) \text { in } B^{i}(\epsilon / 4) \backslash T_{\epsilon i}, \\
& \vec{v}(x) \text { in } Y_{\epsilon i} \backslash B^{i}(\epsilon / 4),
\end{aligned}
$$

where

$x_{\epsilon i}$ is the center of the ball $B^{i}(\epsilon / 4)$ and the center of $Y_{\epsilon i}$,

$\widehat{\vec{v}}_{\epsilon i}$ is the function associated to $\vec{v}()-.\vec{v}\left(x_{\epsilon i}\right)$ in $B^{i}\left(r_{\epsilon}^{2 / 3}\right)$

by Proposition 2.5

$\phi_{\epsilon i}$ is a cut-off function with support in $B^{i}\left(r_{\epsilon}^{2 / 3}\right)$

and equal to 1 in $B^{i}\left(r_{\epsilon}\right)$ :

$\phi_{\epsilon i}(x)=\phi_{\epsilon}\left(\left|x-x_{\epsilon i}\right|\right)$ with

$\phi_{\epsilon}$ in $C^{\infty}([0,1] ;[0,1])$,

$\phi_{\epsilon}$ has its support in $\left[0, r_{\epsilon}^{2 / 3}\right]$,

$\phi_{\epsilon}$ is equal to 1 in $\left[0, r_{\epsilon}\right]$. 


\section{A. Brillard}

The properties of $\vec{v}_{\epsilon}^{o}$ are summarized in the following proposition.

PROPOSITION 2.6. - For any $\vec{v}$ in $\nu(\Omega)$, let $\vec{v}_{\epsilon}^{o}$ be the function defined by (14). Then, $\vec{v}_{\epsilon}^{o}$ belongs to $V\left(\Omega_{\epsilon}\right)$.

If $\alpha_{N}$ is finite, where $\alpha_{N}$ is given by (13), then

$\left(P^{\epsilon} \vec{v}_{\epsilon}^{o}\right)_{\epsilon}$ converges to $\vec{v}$ in the weak topology of $\left(H_{o}^{1}(\Omega)\right)^{N}$. Moreover $\lim _{\epsilon \rightarrow 0} F^{\epsilon}\left(\vec{v}_{\epsilon}^{o}\right)=F(\vec{v})$, where $F$ is the limit functional given by (10).

Let us admit, for a while, these properties and show how (11) may be proved. For a smooth divergence-free function $\vec{v}$ in $\mathcal{V}(\Omega)$, (14) furnishes a test function $\vec{v}_{\epsilon}^{o}$ such that (11) is fulfilled.

If $\vec{v}$ belongs to $V(\Omega)$, choose any sequence $\left(\vec{v}_{n}\right)_{n}$ of smooth divergencefree functions converging to $\vec{v}$ in the strong topology of $\left(H_{o}^{1}(\Omega)\right)^{N}$. For every $n$, (14) furnishes a divergence-free function $\left(\vec{v}_{n}\right)_{\epsilon}^{o}$ such that

$$
\begin{aligned}
& \left(P^{\epsilon}\left(\vec{v}_{n}\right)_{\epsilon}^{o}\right) \frac{w-\left(H_{o}^{1}(\Omega)\right)^{N}}{\epsilon \rightarrow 0} \vec{v}_{n}, \\
& \lim _{\epsilon \rightarrow 0} F^{\epsilon}\left(\left(\vec{v}_{n}\right)_{\epsilon}^{o}\right)=F\left(\vec{v}_{n}\right) .
\end{aligned}
$$

Therefore

$$
\varlimsup_{n \rightarrow+\infty} \lim _{\epsilon \rightarrow 0} F^{\epsilon}\left(\left(\vec{v}_{n}\right)_{\epsilon}^{o}\right)=F(\vec{v}),
$$

since $F$ is continuous, for the strong topology of $\left(H_{0}^{1}(\Omega)\right)^{N}$.

The diagonalization argument of Corollary 1.16. of [2] implies the existence of a subsequence $\left(P^{\epsilon}\left(\vec{v}_{n(\epsilon)}\right)_{\epsilon}^{o}\right)_{\epsilon}$ such that

$$
\begin{aligned}
& \left(P^{\epsilon}\left(\vec{v}_{n(\epsilon)}\right)_{\epsilon}^{o}\right) \frac{w-\left(H_{o}^{1}(\Omega)\right)^{N}}{\epsilon \rightarrow 0} \vec{v}, \\
& \varlimsup_{\epsilon \rightarrow 0} F^{\epsilon}\left(\left(\vec{v}_{n(\epsilon)}\right)_{\epsilon}^{o}\right) \leq F(\vec{v}) .
\end{aligned}
$$

(11) is proved, taking $\vec{v}_{\epsilon}^{o}=\left(\vec{v}_{n(\epsilon)}\right)_{\epsilon}^{o}$, for this general divergence-free function $\vec{v}$.

Proof of Proposition 2.6. - A trivial computation proves that $\vec{v}_{\epsilon}^{o}$ belongs to $V\left(\Omega_{\epsilon}\right)$. Notice, moreover, that

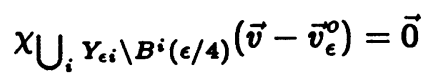

From Lemma 4.1. of [12], one derives that $\left(\chi_{\bigcup_{i} Y_{\epsilon i} \backslash B^{i}(\epsilon / 4)}\right)_{\epsilon}$ converges in the weak topology of $L^{2}(\Omega)$ to the non null constant $\operatorname{Vol}(Y \backslash B(1 / 4))$. Then, 
as soon as $\left(P^{\epsilon} \vec{v}_{\epsilon}^{o}\right)_{\epsilon}$ is bounded in $\left(H_{o}^{1}(\Omega)\right)^{N},(16)$ implies the convergence mentionned in Proposition 2.6. In order to prove that $\left(P^{\epsilon} \vec{v}_{\epsilon}^{o}\right)_{\epsilon}$ is bounded in $\left(H_{o}^{1}(\Omega)\right)^{N}$, we first deduce from the estimates of Proposition 2.5. the convergence

$$
\sum_{i} \operatorname{curl}\left(\phi_{\epsilon i} \widehat{\vec{v}}_{\epsilon i}\right) \underset{\epsilon \rightarrow 0}{\stackrel{s-\left(H^{1}(\Omega)\right)^{N}}{\longrightarrow}} \overrightarrow{0}
$$

(notice that this term guarantees that $\vec{v}_{\epsilon}^{o}$ belongs to $\left.\left(H_{0}^{1}\left(\Omega_{\epsilon}\right)\right)^{N}\right)$. Then we use the properties of the solution $\vec{w}_{\epsilon}^{k}$ given in Proposition A.1. (see the Appendix).

From the convergence (17), we derive

$$
\begin{aligned}
F^{\epsilon}\left(\vec{v}_{\epsilon}^{o}\right)= & \frac{1}{2} \int_{\Omega}|\operatorname{grad} \vec{v}|^{2} d x+ \\
& +\frac{1}{2} \sum_{i} \sum_{k \ell} v_{k}\left(x_{\epsilon i}\right) v_{\ell}\left(x_{\epsilon i}\right) \int_{B^{i}(\epsilon / 4)} \operatorname{grad} \vec{w}_{\epsilon}^{k} \cdot \operatorname{grad} \vec{w}_{\epsilon}^{\ell} d x+o_{\epsilon}
\end{aligned}
$$

with $o_{\epsilon} \underset{\epsilon \rightarrow 0}{\longrightarrow} 0$.

Thanks to the regularity of $\vec{v}$, one derives

$$
\begin{aligned}
F^{\epsilon}\left(\vec{v}_{\epsilon}^{o}\right)=\frac{1}{2} \int_{\Omega}|\operatorname{grad} \vec{v}|^{2} d x+ \\
\quad+\frac{1}{2} \sum_{k \ell} \int_{\Omega} v_{k} v_{\ell} d x\left(\frac{1}{\epsilon^{N}} \int_{B(\epsilon / 4)} \operatorname{grad} \vec{w}_{\epsilon}^{k} \cdot \operatorname{grad} \vec{w}_{\epsilon}^{\ell} d x\right)+o_{\epsilon},
\end{aligned}
$$

and Proposition 2.6. is proved.

$2^{\text {nd }}$ step : Verification of (12) when $\alpha_{N}$ is finite.

Let us first study the case : $T=B(1)$.

Proposition 2.7. - Suppose that $T$ is equal to $B(1)$ and $\alpha_{N}$ is finite. Then, there exists a constant $C$ such that, for every $\vec{v}$ in $\nu(\Omega)$, for every $\vec{u}$ in $V(\Omega)$ and for every sequence $\left(P^{\epsilon} \vec{u}_{\epsilon}\right)_{\epsilon}$ converging to $\vec{u}$ in the weak topology of $\left(H_{o}^{1}(\Omega)\right)^{N}$, with $\vec{u}_{\epsilon}$ in $V\left(\Omega_{\epsilon}\right)$

$$
\begin{aligned}
\varliminf_{\epsilon \rightarrow 0} \int_{\Omega_{\epsilon}} \operatorname{grad} \vec{v}_{\epsilon}^{\circ} \cdot \operatorname{grad} \vec{u}_{\epsilon} d x \geq \int_{\Omega} \operatorname{grad} \vec{v} \cdot \operatorname{grad} \vec{u} d x- \\
\\
\quad-C\|\vec{v}\|_{\left(L^{2}(\Omega)\right)^{N}\|\vec{u}\|_{\left(L^{2}(\Omega)\right)^{N}}}
\end{aligned}
$$

(12) is a trivial consequence of Proposition 2.7. Indeed, choose $\vec{v}$ in $(V(\Omega)$, a sequence $\left(\vec{v}_{n}\right)_{n}$ of smooth functions in $\mathcal{V}(\Omega)$, converging to $\vec{v}$ in the strong 


\section{A. Brillard}

topology of $\left(H_{o}^{1}(\Omega)\right)^{N}$ and $\left(P^{\epsilon} \vec{v}_{\epsilon}\right)_{\epsilon}$ a sequence converging to $\vec{v}$ in the weak topology of $\left(H_{o}^{1}(\Omega)\right)^{N}$, with $\vec{v}_{\epsilon}$ in $V\left(\Omega_{\epsilon}\right)$.

$$
F^{\epsilon}\left(\vec{v}_{\epsilon}\right) \geq F^{\epsilon}\left(\left(\vec{v}_{n}\right)_{\epsilon}^{o}\right)+<\partial F^{\epsilon}\left(\left(\vec{v}_{n}\right)_{\epsilon}^{o}\right), \vec{v}_{\epsilon}-\left(\vec{v}_{n}\right)_{\epsilon}^{o}>
$$

where $\left(\vec{v}_{n}\right)_{\epsilon}^{o}$ is associated to $\vec{v}_{n}$ by $(14)$ and

$$
\left\langle\partial F^{\epsilon}\left(\left(\vec{v}_{n}\right)_{\epsilon}^{o}\right), \vec{v}_{\epsilon}-\left(\vec{v}_{n}\right)_{\epsilon}^{o}>=\int_{\Omega_{\epsilon}} \operatorname{grad}\left(\left(\vec{v}_{n}\right)_{\epsilon}^{o}\right) \cdot \operatorname{grad}\left(\vec{v}_{\epsilon}-\left(\vec{v}_{n}\right)_{\epsilon}^{o}\right) d x\right.
$$

Proposition 2.6. and Proposition 2.7. imply

$$
\begin{gathered}
\varliminf_{\epsilon \rightarrow 0} F^{\epsilon}\left(\vec{v}_{\epsilon}\right) \geq F\left(\vec{v}_{n}\right)+\int_{\Omega} \operatorname{grad} \vec{v}_{n} \cdot \operatorname{grad}\left(\vec{v}-\vec{v}_{n}\right) d x- \\
-C\left\|\vec{v}_{n}\right\|_{\left(L^{2}(\Omega)\right)^{N}\left\|\vec{v}-\vec{v}_{n}\right\|_{\left(L^{2}(\Omega)\right)^{N}}}
\end{gathered}
$$

Let $n$ go to $+\infty:(12)$ is proved.

Proof of Proposition 2.7. - Thanks to (17), one proves

$$
\begin{array}{r}
\int_{\Omega_{\epsilon}} \operatorname{grad} \vec{v}_{\epsilon}^{o} \cdot \operatorname{grad} \vec{u}_{\epsilon} d x=\int_{\Omega} \operatorname{grad} \vec{v} \cdot \operatorname{grad} \vec{u} d x+\sum_{i} \sum_{k} v_{k}\left(x_{\epsilon i}\right) \times \\
\times \int_{B^{i}(\epsilon / 4)} \operatorname{grad} \vec{w}_{\epsilon}^{k} \cdot \operatorname{grad} \vec{u}_{\epsilon} d x+o_{\epsilon}
\end{array}
$$

with $\lim _{\epsilon \rightarrow 0} o_{\epsilon}=0$, and, in fact

$$
\begin{array}{r}
\int_{\Omega_{\epsilon}} \operatorname{grad} \vec{v}_{\epsilon}^{o} \cdot \operatorname{grad} \vec{u}_{\epsilon} d x=\int_{\Omega} \operatorname{grad} \vec{v} \cdot \operatorname{grad} \vec{u} d x+\sum_{i} \sum_{k} v_{k}\left(x_{\epsilon i}\right) \times \\
\times \int_{\partial B^{i}(\epsilon / 4)}\left(\frac{\partial \vec{w}_{\epsilon}^{k}}{\partial \nu}-q_{\epsilon}^{k} \vec{\nu}\right) \vec{u}_{\epsilon} d \sigma_{\epsilon}(x)+o_{\epsilon}
\end{array}
$$

where $\vec{\nu}$ is the outer normal to $\partial B^{i}(\epsilon / 4)$. In order to pass to the limit in the last term of the preceding equality (18), we first use an idea suggested to me by H.Attouch and based on the following interpolation Lemma, whose proof is immediate.

LEMMA 2.8. - For every function $v$ in $C^{1}(\bar{\Omega})$, there exists a function $v_{\epsilon}^{*}$ in $C^{1}(\bar{\Omega})$ (at least) such that

$$
v_{\epsilon}^{*}(x)=v\left(x_{\epsilon i}\right) \text { in } B^{i}(\epsilon / 4) \text { for every } i=1 \text { to } I(\epsilon)
$$




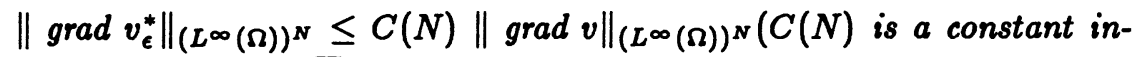
dependant of $v$ in $C^{1}(\bar{\Omega})$ and $\epsilon$ but depending on the dimension $\left.N\right)$,

$\left(v_{\epsilon}^{*}\right)_{\epsilon}$ converges in the strong topology of $L^{2}(\Omega)$ to $v$.

Hence (18) may be written in the following form

$$
\begin{aligned}
\int_{\Omega_{\epsilon}} \operatorname{grad} \vec{v}_{\epsilon}^{o} \cdot \operatorname{grad} \vec{u}_{\epsilon} d x=\int_{\Omega} \operatorname{grad} \vec{v} \cdot \operatorname{grad} \vec{u} d x+ \\
+\sum_{i} \sum_{k} \int_{\partial B^{i}(\epsilon / 4)}\left(\frac{\partial \vec{w}_{\epsilon}^{k}}{\partial \nu}-q_{\epsilon}^{k} \vec{\nu}\right) \cdot \vec{u}_{\epsilon}\left(v_{k}\right)_{\epsilon}^{*} d \sigma_{\epsilon}(x)+o_{\epsilon},
\end{aligned}
$$

where $\left(v_{k}\right)_{\epsilon}^{*}$ is associated to $v_{k}$ by Lemma 2.8 .

Therefore, Proposition 2.7. is a consequence of the convergences mentionned in Lemma 2.8. and Proposition A.1.

Remark 2.9. - The proof of Theorem 2.2., which is actually complete for the case $T=B(1)$ (and $\alpha_{N}$ finite), is partially based on the convergence of the term

$$
\left.\sum_{i}\left(\frac{\partial \vec{w}_{\epsilon}^{k}}{\partial \nu}-q_{\epsilon}^{k} \vec{\nu}\right)\right|_{\partial B^{i}(\epsilon / 4)}
$$

for the strong topology of $\left(H^{-1}(\Omega)\right)^{N}$. This convergence is obtained in the Appendix thanks to the explicit computation of the solution $\left(\vec{w}_{\epsilon}^{k}, q_{\epsilon}^{k}\right)$ of (4). In the study of the asymptotic behaviour of the solution $u_{\epsilon}$ of the Laplace problem [5],

$$
\mid \begin{aligned}
& -\Delta u_{\epsilon}=f \text { in } \Omega_{\epsilon} \\
& u_{\epsilon} \in H_{o}^{1}\left(\Omega_{\epsilon}\right)
\end{aligned}
$$

the convergence, in the strong topology of $H^{-1}(\Omega)$, of the boundary term $\Sigma_{i} \partial w_{\epsilon} /\left.\partial \nu\right|_{\partial B^{i}(\epsilon / 4)}$, where $w_{\epsilon}$ is the solution of the corresponding local problem

$$
\mid \begin{array}{ll}
-\Delta w_{\epsilon}=0 & \text { in } B(\epsilon / 4) \\
w_{\epsilon}=0 & \text { on } \partial T_{\epsilon} \\
w_{\epsilon}=1 & \text { on } \partial B(\epsilon / 4)
\end{array}
$$

was proved for any smooth subset $T$ of $B(1)$.

The case of a general inclusion $T$ was deduced of the case $T=B(1)$, through a maximum principle argument and Lemma 2.8. of [5]. 


\section{A. Brillard}

In the present case of the Stokes system, a maximum principle seems unavailable. This is the reason why, for a general inclusion $T$, we have to slightly modify the test function $\vec{v}_{\epsilon}^{\circ}$.

Let us introduce for $\vec{v}$ in $\mathcal{V}(\Omega)$, the function $\vec{v}_{\epsilon}^{1}$

$$
\vec{v}_{\epsilon}^{1}(x)=\mid \begin{array}{lc}
\vec{v}(x)-\sum_{k} \operatorname{curl}\left(\widehat{\vec{w}}_{\epsilon, i}^{k} \psi_{\epsilon i}\right)(x) v_{k}\left(x_{\epsilon i}\right)-\operatorname{curl}\left(\widehat{\vec{v}}_{\epsilon i} \phi_{\epsilon i}\right)(x) \\
\text { in } B^{i}(\epsilon / 4) \backslash T_{\epsilon i},
\end{array}
$$

where $\psi_{\epsilon i}$ is a cut-off function with support in $B^{i}\left(\frac{\epsilon}{4}-\frac{r_{\epsilon}}{2}\right)$ and equal to 1 in $B^{i}\left(\frac{\epsilon}{8}-\frac{r_{c}}{2}\right)$,

$$
\begin{aligned}
\psi_{\epsilon i}(x)=\psi\left(\frac{\left|x-x_{\epsilon i}\right|+\frac{r_{\epsilon}}{2}}{\frac{\epsilon}{2}}\right) \text { with } & \psi \text { in } C^{\infty}([0,1] ;[0,1]), \\
& \psi \text { has its support in }[0,1 / 2] \text { and } \\
& \psi \text { is equal to } 1 \text { in }[0,1 / 4],
\end{aligned}
$$

$\widehat{\vec{w}}_{\epsilon i}^{k}$ is the function associated to $\vec{e}_{k}-\vec{w}_{\epsilon}^{k}$ in $B^{i}\left(\frac{\epsilon}{4}-\frac{r_{\epsilon}}{2}\right)$ by Proposition 2.5.

$\vec{v}_{\epsilon}^{1}$ is a slight modification of $\vec{v}_{\epsilon}^{o}$ in the sense that

$$
\text { PROPOSITION 2.10. - } \vec{v}_{\epsilon}^{1} \text { belongs to } V\left(\Omega_{\epsilon}\right) \text {. }
$$

Suppose that $\alpha_{N}$ is finite. Then for every $\vec{v}$ in $\nu(\Omega)$

$$
\begin{aligned}
& \left(P^{\epsilon} \vec{v}_{\epsilon}^{1}\right)_{\epsilon} \text { converges, in the weak topology of }\left(H_{o}^{1}(\Omega)\right)^{N}, \text { to } \vec{v}, \\
& \lim _{\epsilon \rightarrow 0} F^{\epsilon}\left(P^{\epsilon} \vec{v}_{\epsilon}^{1}\right)=F(\vec{v}) .
\end{aligned}
$$

There exists a constant $C$ such that for every $\vec{u}$ in $V(\Omega)$ and every sequence $\left(P^{\epsilon} \vec{u}_{\epsilon}\right)_{\epsilon}$ converging to $\vec{u}$ in the weak topology of $\left(H_{o}^{1}(\Omega)\right)^{N}$, with $\vec{u}_{\epsilon}$ in $V\left(\Omega_{\epsilon}\right)$

$$
\begin{aligned}
\varliminf_{\epsilon \rightarrow 0} \int_{\Omega_{e}} \operatorname{grad} \vec{v}_{\epsilon}^{1} \cdot \operatorname{grad} \vec{u}_{\epsilon} d x \geq \int_{\Omega} \operatorname{grad} \vec{v} \cdot \operatorname{grad} \vec{u} d x- \\
\\
\quad-C\|\vec{v}\|_{\left(L^{2}(\Omega)\right)^{N}\|\vec{u}\|_{\left(L^{2}(\Omega)\right)^{N}}}
\end{aligned}
$$

The proof of (12) when $T$ is a subset of $B(1)$ is easily deduced of Proposition 2.10. 
Proof of Proposition 2.10 . - The first three assertions of Proposition 2.10. may be proved in the same way as the corresponding assertions of Proposition 2.6.

Let us prove the last assertion concerning $\vec{v}_{\epsilon}^{\mathbf{1}}$.

$$
\begin{aligned}
& \int_{\Omega_{\epsilon}} \operatorname{grad} \vec{v}_{\epsilon}^{1} \cdot \operatorname{grad} \vec{u}_{\epsilon} d x=\int_{\Omega} \operatorname{grad} \vec{v} \cdot \operatorname{grad} \vec{u} d x+ \\
&+\sum_{i} \sum_{k} v_{k}\left(x_{\epsilon i}\right) \int_{B^{i}(\epsilon / 4)} \operatorname{grad}\left(\operatorname{curl} \widehat{\vec{w}}_{\epsilon i}^{k} \psi_{\epsilon i}\right) . \\
& \cdot \operatorname{grad} \vec{u}_{\epsilon} d x+o_{\epsilon},
\end{aligned}
$$

with $\lim _{\epsilon \rightarrow 0} o_{\epsilon}=0$ (17).

The original point in this proof is to pass to the limit in the last term of the preceding equality. Denote $A_{\epsilon}$ this term, which may be written, thanks to (4) and the estimates of Proposition 2.5. as

$$
\begin{aligned}
A_{\epsilon}=\sum_{k} \sum_{i} v_{k}\left(x_{\epsilon i}\right) \int_{C_{\epsilon i}} q_{\epsilon}^{k} \operatorname{grad} \psi_{\epsilon i} \vec{u}_{\epsilon} d x- \\
-\sum_{k} \sum_{i} v_{k}\left(x_{\epsilon i}\right) \int_{C_{\epsilon i}} \Delta \psi_{\epsilon i}\left(\vec{e}_{k}-\vec{w}_{\epsilon}^{k}\right) \vec{u}_{\epsilon} d x- \\
-\sum_{k} \sum_{i} v_{k}\left(x_{\epsilon i}\right) \int_{C_{\epsilon i}} \operatorname{grad}\left(\vec{e}_{k}-\vec{w}_{\epsilon}^{k}\right) \operatorname{grad} \psi_{\epsilon i} \vec{u}_{\epsilon} d x+o_{\epsilon},
\end{aligned}
$$

where $C_{\epsilon i}$ is the set $B^{i}\left(\frac{\epsilon}{4}-\frac{r_{c}}{2}\right) \backslash B^{i}\left(\frac{\epsilon}{8}-\frac{r_{\epsilon}}{2}\right)$.

The pointwise estimates obtained by Marchenko and Hrouslov on $\vec{w}_{\epsilon}^{k}, \vec{q}_{\epsilon}^{k}$ and the derivatives of $\vec{w}_{\epsilon}^{k}$ (see Proposition A.1.) imply the existence of a constant $C$ independant of $\epsilon, \vec{v}$ and $\vec{u}$ such that

$$
\varlimsup_{\epsilon \rightarrow 0}\left|A_{\epsilon}\right| \leq C\|\vec{v}\|_{\left(L^{2}(\Omega)\right)^{N}}\|\vec{u}\|_{\left(L^{2}(\Omega)\right)^{N}}
$$

and Proposition 2.10. is proved.

$3^{\text {rd }}$ step $-\alpha_{n}=+\infty$.

For every $\vec{v}$ in $\left(H_{o}^{1}(\Omega)\right)^{N}$, one observes that

$$
\begin{gathered}
F^{\epsilon}(\vec{v}) \geq F_{K}^{\epsilon}(\vec{v}), \\
-241-
\end{gathered}
$$




\section{A. Brillard}

where $F_{K}^{\epsilon}$ is the functional defined by (9) in the case $\alpha_{N}=K$ and $T=B(1)$. Therefore, for every $K$ in $R^{+}$and for every $\vec{v}$ in $\left(H_{0}^{1}(\Omega)\right)^{N}$

$$
\left(\tau-\varlimsup_{\epsilon \rightarrow 0} F^{\epsilon}\right)(\vec{v}) \geq\left(\tau-\varliminf_{\epsilon \rightarrow 0} F^{\epsilon}\right)(\vec{v}) \geq\left(\tau-\varliminf_{\epsilon \rightarrow 0} F_{K}^{\epsilon}\right)(\vec{v})
$$

$$
\left(\tau=w-\left(H_{0}^{1}(\Omega)\right)^{N}\right)
$$

and for every $\vec{v}$ in $V(\Omega)$

$$
\begin{aligned}
\left(\tau-\varliminf_{\epsilon \rightarrow 0} F^{\epsilon}\right)(\vec{v}) & \geq \sup _{K}\left(\tau-\varliminf_{\epsilon \rightarrow 0} F_{K}^{\epsilon}\right)(\vec{v}) \\
& \geq \sup _{K}\left\{\frac{1}{2} \int_{\Omega}|\operatorname{grad} \vec{v}|^{2} d x+\frac{1}{2} \sum_{k \ell} \int_{\Omega}\left(M_{K}\right)_{k \ell} v_{k} v_{\ell} d x\right\} .
\end{aligned}
$$

From Remark 2.3. one derives

$$
\left(\tau-\varliminf_{\epsilon \rightarrow 0} F^{\epsilon}\right)(\vec{v}) \geq \sup _{k}\left\{\frac{1}{2} \int_{\Omega}|\operatorname{grad} \vec{v}|^{2} d x+\frac{K}{2} \sum_{k \ell} \int_{\Omega}\left(M_{1}\right)_{k \ell} v_{k} v_{\ell} d x\right\} .
$$

If $\vec{v}$ is equal to $\overrightarrow{0}$, then

$$
\left(\tau-\varlimsup_{\epsilon \rightarrow 0} F^{\epsilon}\right)(\overrightarrow{0})=\left(\tau-\varliminf_{\epsilon \rightarrow 0} F^{\epsilon}\right)(\overrightarrow{0})=0 .
$$

It $\vec{v}$ is different from $\overrightarrow{0}$ (almost everywhere) then

$$
\left(\tau-\varlimsup_{\epsilon \rightarrow 0} F^{\epsilon}\right)(\vec{v}) \geq\left(\tau-\varliminf_{\epsilon \rightarrow 0} F^{\epsilon}\right)(\vec{v})=+\infty
$$

Remark 2.11. - The convergence of $\left(P^{\epsilon} \vec{u}_{\epsilon}\right)_{\epsilon}$ to the solution $\vec{u}_{o}$ of (2), may be obtained without epi-convergence methods. For $\vec{v}$ in $\mathcal{V}(\Omega)$ and $\alpha_{N}$ finite, one immediately proves

$$
\begin{aligned}
\lim _{\epsilon \rightarrow 0}\left(\int_{\Omega_{\epsilon}} \operatorname{grad} \vec{u}_{\epsilon} \cdot \operatorname{grad} \vec{v}_{\epsilon}^{\circ} d x-\int_{\Omega_{\epsilon}} \vec{f} \cdot \vec{v}_{\epsilon}^{\circ} d x\right) & =\int_{\Omega} \operatorname{grad} \vec{u}_{o} \cdot \operatorname{grad} \vec{v} d x+ \\
& +\sum_{k \ell} \int_{\Omega} M_{k \ell}\left(\vec{u}_{o}\right)_{k} v_{\ell} d x-\int_{\Omega} \vec{f} \cdot \vec{v} d x
\end{aligned}
$$

$$
\begin{aligned}
& \left(\text { or } \lim _{\epsilon \rightarrow 0}\left(\int_{\Omega_{\epsilon}} \operatorname{grad} \vec{u}_{\epsilon} \cdot \operatorname{grad} \vec{v}_{\epsilon}^{1} d x-\int_{\Omega_{\epsilon}} \vec{f} \cdot \vec{v}_{\epsilon}^{1} d x\right)=\int_{\Omega} \operatorname{grad} \vec{u}_{o} \cdot \operatorname{grad} \vec{v} d x+\right. \\
& \left.+\sum_{k \ell} \int_{\Omega} M_{k \ell}\left(\vec{u}_{o}\right)_{k} v_{\ell} d x-\int_{\Omega} \vec{f} \cdot \vec{v} d x\right)
\end{aligned}
$$


where $\vec{v}_{\epsilon}^{o}$ (resp. $\vec{v}_{\epsilon}^{1}$ ) is associated to $\vec{v}$ by (14) (resp. (19)).

However, we pointed out, in the introduction, the advantage of epiconvergence methods.

Remark 2.12. - In the constructions (14) or (19), the values $v_{k}\left(x_{\epsilon i}\right)$ (or $\left.\vec{v}\left(x_{\epsilon i}\right)\right)$ may be changed into

$$
\frac{1}{\operatorname{Vol}(B(\epsilon / 4))} \int_{B^{i}(\epsilon / 4)} v_{k}(x) d x\left(\text { or } \frac{1}{\operatorname{Vol}(B(\epsilon / 4))} \int_{B^{i}(\epsilon / 4)} \vec{v}(x) d x\right) .
$$

\section{$\S$ III. Convergence of the internal pressure}

When $r_{\epsilon}=\lambda \epsilon(0<\lambda<1 / 2)$, Tartar proved in [13] the following result :

THEOREM 3.1. - There exists a linear operator $R^{\epsilon}$ from $\left(H_{o}^{1}(\Omega)\right)^{N}$ into $\left(H_{o}^{1}\left(\Omega_{\epsilon}\right)\right)^{N}$, such that :

R1) For every $\vec{v}$ in $\left(H_{o}^{1}\left(\Omega_{\epsilon}\right)\right)^{N}, R^{\epsilon} P^{\epsilon} \vec{v}=\vec{v}$,

R2) For every $\vec{v}$ in $V(\Omega), R^{\epsilon} \vec{v}$ belongs to $V\left(\Omega_{\epsilon}\right)$,

RS) There exists a strictly positive constant $C$ such that for every $\vec{v}$ in $\left(H_{o}^{1}(\Omega)\right)^{N}$

$$
\begin{aligned}
& \left\|R^{\epsilon} \vec{v}\right\|_{\left(L^{2}\left(\Omega_{\epsilon}\right)\right)^{N}} \leq C\|\vec{v}\|_{\left(L^{2}(\Omega)\right)^{N}}+C \epsilon\|\operatorname{grad} \vec{v}\|_{\left(L^{2}(\Omega)\right)^{N^{2}}} \\
& \left\|\operatorname{grad} R^{\epsilon} \vec{v}\right\|_{\left(L^{2}(\Omega)\right)^{N^{2}}} \leq \frac{C}{\epsilon}\|\vec{v}\|_{\left(L^{2}(\Omega)\right)^{N}}+C\|\operatorname{grad} \vec{v}\|_{\left(L^{2}(\Omega)\right)^{N^{2}}} .
\end{aligned}
$$

Let $\left(\vec{u}_{\epsilon}, p_{\epsilon}\right)$ the solution of the Stokes problem (1) with $r_{\epsilon}=\lambda \epsilon$.

Let $\bar{p}_{\epsilon}$ be the element of $L^{2}(\Omega) / R$ such that for every $\vec{v}$ in $\left(H_{o}^{1}(\Omega)\right)^{N}$, $<\operatorname{grad} \bar{p}_{\epsilon}, \vec{v}>=<\operatorname{grad} p_{\epsilon}, R^{\epsilon} \vec{v}>$ (this is justified by $\left.\left.R 2\right)\right)$. Then $\left(\operatorname{grad} \bar{p}_{\epsilon}\right)_{\epsilon}$ converges in the strong topology of $\left(H^{-1}(\Omega)\right)^{N}$ to $\operatorname{grad} p_{o}$ and $\left(\bar{p}_{\epsilon}\right)_{\epsilon}$ converges in the strong topology of $L^{2}(\Omega) / R$ to $p_{0}$.

Notice that for every $\vec{v}$ in $\left(H_{o}^{1}(\Omega)\right)^{N}$ :

$$
<R^{\epsilon *}\left(\operatorname{grad} p_{\epsilon}\right), \vec{v}>=<\operatorname{grad} \bar{p}_{\epsilon}, \vec{v}>\underset{\epsilon \rightarrow 0}{\longrightarrow}<\operatorname{grad} p_{o}, \vec{v}>,
$$

In this case $R^{\epsilon *}\left(\operatorname{grad} p_{\epsilon}\right)$ is an extension of $\left.\operatorname{grad} p_{\epsilon}(\mathrm{R} 1)\right)$ and is still a gradient! The construction of $R^{\epsilon}$, given in [13], Lemma 3, is made through the change of scale $x=\epsilon y$, and then requires the equality $r_{\epsilon}=\lambda \epsilon$, for some $\lambda$ in $] 0,1 / 2[$. 


\section{A. Brillard}

In the present case, that is when $\lim _{\epsilon \rightarrow o}\left(r_{\epsilon} / \epsilon\right)=0$, such a construction seems impossible. Nevertheless, the purpose of this paragraph is to present two restriction operators $R_{j}^{\epsilon}$, defined on two subspaces $X_{j}$ of $\left(H_{o}^{1}(\Omega)\right)^{N}$, consisting of sufficiently smooth functions in $\left(H_{o}^{1}(\Omega)\right)^{N}$, such that for every $\vec{v}$ in $X_{j}$ :

$$
\lim _{\epsilon \rightarrow 0}<\operatorname{grad} p_{\epsilon}, R_{j}^{\epsilon} \vec{v}>=\left(\lim _{\epsilon \rightarrow 0}<R_{j}^{\epsilon *}\left(\operatorname{grad} p_{\epsilon}\right), \vec{v}>\right)=<\operatorname{grad} p_{o}, \vec{v}>
$$

A) $T$ is equal to $B(1), X_{1}=\left(H_{o}^{1}(\Omega)\right)^{N} \cap\left(C^{1}(\bar{\Omega})\right)^{N}, \alpha_{N}$ is finite

One of the main difficulties in the construction of the test function $\vec{v}_{\epsilon}^{o}$ (14), was to preserve the divergence-free condition. In the present study, this condition has no more to be satisfied. A first idea is to simplify the last term of (14) and will be used later on. But, following Murat's sugestion, [11], a simpler expresssion may be used.

THEOREM 3.2. - Let $R_{1}^{\epsilon}$ be the linear operator from $X_{1}$ into $\left(H_{o}^{1}\left(\Omega_{\epsilon}\right)\right)^{N}$ defined by

$$
\forall \vec{v} \in X_{1} \quad R_{1}^{\epsilon} \vec{v}(x)=\sum_{k} \vec{w}_{\epsilon}^{k}(x) v_{k}(x) .
$$

Then,

a) for every $\vec{v}$ in $\left(H_{o}^{1}(\Omega)\right)^{N}$

$$
R_{1}^{\epsilon} \vec{v} \underset{\epsilon \rightarrow 0}{w-\left(H_{o}^{1}(\Omega)\right)^{N}} \vec{v}
$$

b) for every $\vec{v}$ in $X_{1}$ :

$$
<\operatorname{grad} p_{\epsilon}, R_{1}^{\epsilon} \vec{v}>\underset{\epsilon \rightarrow 0}{\longrightarrow}<\operatorname{grad} p_{o}, \vec{v}>
$$

Proof of Theorem 9.2.

a) is a consequence of Proposition A.1.

b)

$$
\begin{aligned}
<\operatorname{grad} p_{\epsilon}, R_{1}^{\epsilon} \vec{v}>= & \int_{\Omega_{\epsilon}} \operatorname{grad} \vec{u}_{\epsilon} \cdot \operatorname{grad}\left(\sum_{k} \vec{w}_{\epsilon}^{k} v_{k}\right) d x+ \\
& +\int_{\Omega_{\epsilon}} \vec{f} \cdot\left(\sum_{k} \vec{w}_{\epsilon}^{k} v_{k}\right) d x \\
& -244-
\end{aligned}
$$




\section{Asymptotic analysis of incompressible}

An integration by parts in the right term of the preceding equality implies using (4)

$$
\begin{aligned}
& <\operatorname{grad} p_{\epsilon}, R_{1}^{\epsilon} \vec{v}>=-\sum_{i} \sum_{k} \int_{B^{i}(\epsilon / 4)} q_{\epsilon}^{k} \vec{u}_{\epsilon} \operatorname{grad} v_{k} d x+ \\
& +\sum_{i} \sum_{k} \int_{\partial B^{i}(\epsilon / 4)}\left(\frac{\partial \vec{w}_{\epsilon}^{k}}{\partial \nu}-q_{\epsilon}^{k} \vec{\nu}\right) \vec{u}_{\epsilon} v_{k} d \sigma_{\epsilon}(x)+ \\
& +\sum_{k} \int_{\Omega_{\epsilon}} \operatorname{grad} \vec{u}_{\epsilon} \operatorname{grad} v_{k} \vec{w}_{\epsilon}^{k} d x-\sum_{k} \int_{\Omega_{\epsilon}} \operatorname{grad} \vec{w}_{\epsilon}^{k} \operatorname{grad} v_{k} \vec{u}_{\epsilon} d x+ \\
& +\sum_{k} \int_{\Omega_{\epsilon}} \vec{f} \cdot \vec{w}_{\epsilon}^{k} v_{k} d x .
\end{aligned}
$$

An appropriate choice of $q_{\epsilon}^{k}$ (so that $\left.\chi_{\cup_{i} B^{i}(\epsilon / 4) \backslash T_{\epsilon i}} q_{\epsilon}^{k}\right)_{\epsilon}$ converges to 0 , in the weak topology of $L^{2}(\Omega)$ (see Lemma A.4)), Theorem 2.2 and Proposition A.1 imply

$$
\begin{aligned}
\lim _{\epsilon \rightarrow 0}<\operatorname{grad} p_{\epsilon}, R_{1}^{\epsilon} \vec{v}> & =\int_{\Omega} \operatorname{grad} \vec{u}_{o} \cdot \operatorname{grad} \vec{v} d x+\sum_{k \ell} \int_{\Omega} M_{k \ell}\left(\vec{u}_{o}\right)_{k} v_{\ell} d x- \\
& -\int_{\Omega} \vec{f} \cdot \vec{v} d x \\
& =<\operatorname{grad} p_{o}, \vec{v}>.
\end{aligned}
$$

Remark 3.3. - 1) In the construction (21) of $R_{1}^{\epsilon}$, the preservation of the divergence-free condition is violated. Indeed, the following choice of space and restriction operator preserves this condition

$$
\begin{aligned}
& X_{2}=\left\{\vec{v} \in X_{1} / \operatorname{proj}_{V(\Omega)} \vec{v} \text { belongs to } X_{1}\right\}, \\
& R_{2}^{\epsilon} \vec{v}=R_{1}^{\epsilon}\left(\operatorname{proj}_{V(\Omega)} \vec{v}\right)+\left(\operatorname{proj}_{V(\Omega)^{\perp}}(\vec{v})\right)_{\epsilon}^{o} .
\end{aligned}
$$

where proj denotes the usual projection on a closed subspace of a Hilbert space. Nevertheless, from a physical viewpoint, it seems unnecessary to extend grad $p_{\epsilon}$ into a gradient (or something close to a gradient) since the fluid fills $\boldsymbol{\Omega}_{\epsilon}$ only.

2) In fact, from the construction (21) of the restriction operator $R_{1}^{\epsilon}$, one obtains the following result [11] :

If there exists $\bar{p}_{\epsilon}$ in $L^{2}(\Omega) / R$ such that $\bar{p}_{\epsilon \mid \Omega_{\epsilon}}=p_{\epsilon}\left(\right.$ in $\left.L^{2}(\Omega) / R\right)$, and $\left(\bar{p}_{\epsilon}\right)_{\epsilon}$ is bounded in $L^{2}(\Omega) / R$, then $\left(\bar{p}_{\epsilon}\right)_{\epsilon}$ converges to $p_{o}$ in the weak topology of $L^{2}(\Omega) / R$. 


\section{A. Brillard}

However, we did not yet obtain such uniform estimates on the pressure $p_{\epsilon}$.

B) $T$ is the ball $B(1), X_{3}=\left(H_{o}^{1}(\Omega)\right)^{N} \cap\left(C^{o}(\bar{\Omega})\right)^{N}$ and $\alpha_{N}$ is finite

THEOREM 3.4. - Let $R_{3}^{\epsilon}$ be the linear operator from $X_{3}$ into $\left(H_{o}^{1}\left(\Omega_{\epsilon}\right)\right)^{N}$ defined by

$$
\forall \vec{v} \in X_{3}: R_{3}^{\epsilon} \vec{v}(x)=\mid \begin{gathered}
\vec{v}(x)-\sum_{k}\left(\vec{e}_{k}-\vec{w}_{\epsilon}^{k}(x)\right) v_{k}\left(x_{\epsilon i}\right)- \\
-\left(\vec{v}(x)-\vec{v}\left(x_{\epsilon i}\right)\right) \phi_{\epsilon i}(x) \quad \text { in } B^{i}(\epsilon / 4), \\
\vec{v}(x) \quad \text { in } Y_{\epsilon i} \backslash B^{i}(\epsilon / 4),
\end{gathered}
$$

where $\phi_{\epsilon i}$ is the cut-off function defined in (15) ( $\phi_{\epsilon i}$ has its support in $B^{i}\left(r_{\epsilon}^{2 / 3}\right)$ and is equal to 1 in $\left.B^{i}\left(r_{\epsilon}\right)\right)$.

Then

$$
\forall \vec{v} \in X_{3} \quad<\operatorname{grad} p_{\epsilon}, R_{3}^{\epsilon} \vec{v}>\underset{\epsilon \rightarrow 0}{\longrightarrow}<\operatorname{grad} p_{o}, \vec{v}>
$$

Proof of Theorem 3.4. - Let us first suppose that $\vec{v}$ belongs to $X_{1}$. Then

LEMMA 3.5. - For every $\vec{v}$ in $X_{1}, P^{\epsilon} R_{3}^{\epsilon} \vec{v} \frac{w-\left(H_{o}^{1}(\Omega)\right)^{N}}{\epsilon \rightarrow 0} \vec{v}$.

There exists a quantity $o_{\epsilon}$ which converges to 0 when $\epsilon$ goes to 0 such that for every $\vec{v}$ in $X_{1}$

$$
\left\|\sum_{i}\left(\vec{v}(.)-\vec{v}\left(x_{\epsilon i}\right)\right) \phi_{\epsilon i}\right\|_{\left(H^{1}(\Omega)\right)^{N}} \leq o_{\epsilon}\|\operatorname{grad} \vec{v}\|_{\left(L^{\infty}(\Omega)\right)^{N^{2}}}
$$

Proof of Lemma 3.5. - As in Prop. 2.6., we only need to show that $\left(P^{\epsilon} R_{3}^{\epsilon} \vec{v}\right)_{\epsilon}$ is bounded in $\left(H_{o}^{1}(\Omega)\right)^{N}$. Using the regularity of $\vec{v}$ (in $\left.X_{1}\right)$ and Prop. A.1, we have only to study the last term of (22).

One immediately verifies :

$$
\sum_{i} \int_{B^{i}(\epsilon / 4)}|\operatorname{grad} \vec{v}|^{2}\left(\phi_{\epsilon i}\right)^{2} d x \leq\|\operatorname{grad} \vec{v}\|_{\left(L^{\infty}(\Omega)\right)^{N^{2}}}^{2}\left(\sum_{i} \int_{B^{i}\left(r_{\epsilon}^{2 / 3}\right)} d x\right)
$$

and also

$$
\begin{aligned}
\sum_{i} \int_{B^{i}(\epsilon / 4)}\left|\vec{v}(x)-\vec{v}\left(x_{\epsilon i}\right)\right|^{2}\left|\operatorname{grad} \phi_{\epsilon i}\right|^{2} d x & \leq C\|\operatorname{grad} \vec{v}\|_{\left(L^{\infty}(\Omega)\right)^{N^{2}}}^{2} . \\
& \cdot\left(\sum_{i} \epsilon^{4 / 3} \int_{B^{i}\left(r_{\epsilon}^{2 / 3}\right)}\left|\operatorname{grad} \phi_{\epsilon i}\right|^{2} d x\right) .
\end{aligned}
$$




\section{Asymptotic analysis of incompressible}

A trivial computation of the last term in the preceding inequality ends the proof of Lemma 3.5.

Let us come back to the proof of Theorem 3.4.

If $\vec{v}$ belongs to $X_{1}$, one derives, using (4), Proposition A.1 and Lemma 3.5.

$$
\begin{aligned}
<\operatorname{grad} p_{\epsilon}, R_{3}^{\epsilon} \vec{v}> & =\int_{\Omega_{\epsilon}} \operatorname{grad} \vec{u}_{\epsilon} \operatorname{grad} \vec{v} d x+\int_{\Omega} \vec{f} \cdot \vec{v} d x+ \\
& +\sum_{i} \sum_{k} \int_{\partial B^{i}(\epsilon / 4)}\left(\frac{\partial \vec{w}_{\epsilon}^{k}}{\partial \nu}-q_{\epsilon}^{k} \vec{\nu}\right) \vec{u}_{\epsilon} v_{k}\left(x_{\epsilon i}\right) d \sigma_{\epsilon}(x)+o_{\epsilon}(\vec{v})
\end{aligned}
$$

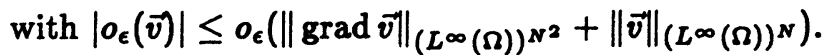

For $\vec{v}$ in $X_{1},(23)$ gives

$$
\begin{aligned}
<\operatorname{grad} p_{\epsilon}, R_{3}^{\epsilon} \vec{v}> & =\int_{\Omega_{\epsilon}} \operatorname{grad} \vec{u}_{\epsilon} \cdot \operatorname{grad} \vec{v} d x+\int_{\Omega} \vec{f} . \vec{v} d x+ \\
& +\sum_{i} \sum_{k} \int_{\partial B^{i}(\epsilon / 4)}\left(\frac{\partial \vec{w}_{\epsilon}^{k}}{\partial \nu}-q_{\epsilon}^{k} \vec{\nu}\right) \vec{u}_{\epsilon} \vec{v}_{\epsilon k} d \sigma_{\epsilon}(x)+o_{\epsilon}(\vec{v}) \\
& \underset{\epsilon \rightarrow 0}{\longrightarrow} \int_{\Omega} \operatorname{grad} \vec{u}_{o} \cdot \operatorname{grad} \vec{v} d x+\int_{\Omega} \vec{f} \cdot \vec{v} d x+ \\
& \quad+\sum_{k} \int_{\Omega} M_{k} \vec{u}_{o} v_{k} d x,
\end{aligned}
$$

where $M_{k}$ is the $k^{\text {th }}$ column vector of the matrix $M(3)$.

If $\vec{v}$ belongs to $X_{3}$, there exists a sequence $\left(\vec{v}_{n}\right)_{n}$ of functions in $X_{1}$ converging to $\vec{v}$ in the strong topology of $\left(H_{o}^{1}(\Omega)\right)^{N}$ and the topology of $\left(L^{\infty}(\Omega)\right)^{N}$. Then

$$
<\operatorname{grad} p_{\epsilon}, R_{3}^{\epsilon} \vec{v}>=<\operatorname{grad} p_{\epsilon}, R_{3}^{\epsilon} \vec{v}_{n}>+<\operatorname{grad} p_{\epsilon}, R_{3}^{\epsilon}\left(\vec{v}-\vec{v}_{n}\right)>,
$$

And one immediately verifies that

$$
\left|<\operatorname{grad} p_{\epsilon}, R_{3}^{\epsilon}\left(\vec{v}-\vec{v}_{n}\right)>\right| \leq C\left(\left\|\vec{v}-\vec{v}_{n}\right\|_{\left(H_{o}^{1}(\Omega)\right)^{N}}+\left\|\vec{v}-\vec{v}_{n}\right\|_{\left.\left(L^{\infty}(\Omega)\right)^{N}\right)} .\right.
$$

C) $T$ is a general subset of $B(1)$ and $\alpha_{N}$ is finite

THEOREM 3.6. - The conclusions of Theorems 9.2 and 9.4 are valid in this case, simply changing (21) or the second term of (22) into

$$
\sum_{k}\left(\vec{e}_{k}-\vec{w}_{\epsilon}^{k}(x)\right) \dot{v}_{k}\left(x_{\epsilon i}\right) \psi_{\epsilon i}(x),
$$

where $\psi_{\epsilon i}$ is the cut-off function defined in (20). 


\section{A. Brillard}

\section{Appendix}

\section{Properties of the solution $\left(\vec{w}_{\epsilon}^{k}, q_{\epsilon}^{k}\right)$ of $(4)$}

The solution $\vec{w}_{\epsilon}^{k}$ of (4) may be extended by $\vec{e}_{k}$ on $Y_{\epsilon} \backslash B(\epsilon / 4)$ and then $\epsilon$-periodically in $R^{N}$. The function obtained in this way will be still denoted by $\vec{w}_{\epsilon}^{k}$. Trivially, the function $\vec{w}_{\epsilon}^{k}$ restricted to $\Omega$ is in $\left(H^{1}(\Omega)\right)^{N}$.

We have summarized in the next Proposition the properties of this (extended) function $\vec{w}_{\epsilon}^{k}$.

Proposition A.1.- a) If $\alpha_{N}$ is finite (see (19)), then the sequence $\left(\vec{w}_{\epsilon}^{k}\right)_{\epsilon}$ converges to $\vec{e}_{k}$ in the weak topology of $\left(H^{1}(\Omega)\right)^{N}$.

b) If $\alpha_{N}$ is finite and if $T$ is equal to $B(1)$, then the sequence

$$
\left(\left.\sum_{i}\left(\frac{\partial \vec{w}_{\epsilon}^{k}}{\partial \nu}-q_{\epsilon}^{k} \vec{\nu}\right)\right|_{\partial B^{i}(\epsilon / 4)}\right)_{\epsilon}
$$

converges in the strong topology of $\left(H^{-1}(\Omega)\right)^{N}$ to the $k^{\text {th }}$ column vector of the matrix $M$ defined by ( $(3)$.

c) [10] If $\alpha_{N}$ is finite but if $T$ is a general model inclusion, then there exists an appropriate choice of $q_{\epsilon}^{k}$ (so that $q_{\epsilon}^{k}$, which is defined up to an additive constant, vanishes at one point (at least) in $C_{\epsilon i}=B^{i}\left(\frac{\epsilon}{4}-\right.$ $\left.\left.\frac{r_{c}}{2}\right) \backslash B^{i}\left(\frac{\epsilon}{8}-\frac{r_{c}}{2}\right)\right)$ such that the following pointwise estimates are true : for every $s_{\epsilon}\left(s_{\epsilon}<\epsilon\right)$ and for every $c(c>0)$, there exists a constant $C$ such that

if $N=3$ : for every $x$ in $B\left(s_{\epsilon}\right)$ verifying : $d\left(x, T_{\epsilon}\right) \geq c r_{\epsilon}$,

$$
\begin{array}{ll}
\left|\vec{e}_{k}-\vec{w}_{\epsilon}^{k}\right|(x) \leq C_{\frac{r_{\epsilon}}{d\left(x, T_{\epsilon}\right)}} & ; \quad\left|D_{i} \vec{w}_{\epsilon}^{k}\right|(x) \leq C_{\frac{r_{\epsilon}}{d\left(x, T_{\epsilon}\right)^{2}}} \\
\left|D_{i} D_{j} \vec{w}_{\epsilon}^{k}\right|(x) \leq C_{\frac{r_{e}}{d\left(x, T_{\epsilon}\right)^{3}}} & ; \quad\left|q_{\epsilon}^{k}\right|(x) \leq C_{\frac{r_{e}}{d\left(x, T_{\epsilon}\right)^{2}}}
\end{array}
$$

if $N=2$ : for every $x$ in $B\left(s_{\epsilon}\right)$ verifying : $d\left(x, T_{\epsilon}\right) \geq \exp \left(-c\left(\left|\log r_{\epsilon}\right|\right)^{1 / 2}\right)$,

$$
\begin{array}{cl}
\left|\vec{e}_{k}-\vec{w}_{\epsilon}^{k}\right|(x) \leq C \frac{\left|\log d\left(x, T_{\epsilon}\right)\right|+1}{\left|\log r_{\epsilon}\right|} & ;\left|D_{i} \vec{w}_{\epsilon}^{k}\right|(x) \leq C_{\left|\log r_{\epsilon}\right| d\left(x, T_{\epsilon}\right)} \\
\left|D_{i} D_{j} \vec{w}_{\epsilon}^{k}\right|(x) \leq C_{\left|\log r_{\epsilon}\right| d\left(x, T_{\epsilon}\right)^{2}} & ;\left|q_{\epsilon}^{k}(x)\right| \leq C_{\left|\log r_{\epsilon}\right| d\left(x, T_{\epsilon}\right)}
\end{array}
$$

Proof of Proposition A.1. - a) From the definition of the (extended) function $\vec{w}_{\epsilon}^{k}$, one derives

$$
\begin{gathered}
\chi_{\cup_{i} Y_{\epsilon i} \backslash B^{i}(\epsilon / 4)}\left(\vec{e}_{k}-\vec{w}_{\epsilon}^{k}\right)=\overrightarrow{0} \text { in } \Omega . \\
-248-
\end{gathered}
$$


Then, as soon as the sequence $\left(\vec{w}_{\epsilon}^{k}\right)_{\epsilon}$ is bounded in. $\left(H^{1}(\Omega)\right)^{N}$, and then relatively compact in the strong topology of $\left(L^{2}(\Omega)\right)^{N}$, the convergence announced in a) is proved, since $\left(\chi_{\cup_{i} Y_{\epsilon i} \backslash B^{i}(\epsilon / 4)}\right)_{\epsilon}$ converges in the weak topology of $L^{2}(\Omega)$ to the non null constant $\operatorname{Vol}(Y \backslash B(1 / 4))$. Moreover, from (4), one deduces

$$
\int_{B(\epsilon / 4) \backslash T_{\epsilon}}\left|\operatorname{grad} \vec{w}_{\epsilon}^{k}\right|^{2} d x \leq \int_{B(\epsilon / 4) \backslash T_{\epsilon}}\left|\operatorname{grad} \vec{w}_{\epsilon}^{k *}\right|^{2} d x,
$$

where $\vec{w}_{\epsilon}^{k *}$ is the solution of the same local problem (4) but for $T=B(1)$. Therefore, we have only to prove that if $T$ is equal to $B(1)$ and if $\alpha_{N}$ is finite, then $\left(\vec{w}_{\epsilon}^{k *}\right)_{\epsilon}$ is bounded in $\left(H^{1}(\Omega)\right)^{N}$. As in [5] (Theorem 2.2.), this is a consequence of the explicit computation of $\vec{w}_{\epsilon}^{k *}$ given in the following

Proposition A.2. - Suppose that $N=3$ and denote by $\rho$ the quantity $\left(x^{2}+y^{2}+z^{2}\right)^{1 / 2}$. Then

$$
\vec{w}_{\epsilon}^{1 *}(x, y, z)=\operatorname{curl}\left(0,-f_{\epsilon}(\rho) z, f_{\epsilon}(\rho) y\right), q_{\epsilon}^{1 *}(x, y, z)=g_{\epsilon}(\rho) x \text { in } B(\epsilon / 4),
$$

with

$$
\begin{aligned}
& f_{\epsilon}(\rho)=A_{\epsilon} \rho^{2}+B_{\epsilon} \rho^{-3}+C_{\epsilon} \rho^{-1}+D_{\epsilon} \\
& g_{\epsilon}(\rho)=20 A_{\epsilon}+2 C_{\epsilon} \rho^{-3},
\end{aligned}
$$

$A_{\epsilon}, B_{\epsilon}, C_{\epsilon}$ and $D_{\epsilon}$ being four positive quantities.

Moreover, $A_{\epsilon}$ is equivalent to $r_{\epsilon} \epsilon^{-3}$ (in the sense that $\epsilon^{3} A_{\epsilon} / r_{\epsilon}$ converges to a constant in $R^{+*}$, when $\epsilon$ goes to 0$), B_{\epsilon}$ is equivalent to $r_{\epsilon}^{3}, C_{\epsilon}$ is equivalent to $r_{\epsilon}$ and $D_{\epsilon}$ is equivalent to 1 . The two solutions $\vec{w}_{\epsilon}^{2 *}$ and $\vec{w}_{\epsilon}^{3 *}$ are given by

$$
\begin{aligned}
& \left(\vec{w}_{\epsilon}^{2 *}\right)_{1}(x, y, z)=\left(\vec{w}_{\epsilon}^{1 *}\right)_{2}(y, x, z) ;\left(\vec{w}_{\epsilon}^{2 *}\right)_{2}(x, y, z)=\left(\vec{w}_{\epsilon}^{1 *}\right)_{1}(y, x, z), \\
& \left(\vec{w}_{\epsilon}^{2 *}\right)_{3}(x, y, z)=\left(\vec{w}_{\epsilon}^{1 *}\right)_{3}(y, x, z) ; q_{\epsilon}^{2 *}(x, y, z)=q_{\epsilon}^{1 *}(y, x, z) \\
& \left(\vec{w}_{\epsilon}^{3 *}\right)_{1}(x, y, z)=\left(\vec{w}_{\epsilon}^{1 *}\right)_{3}(z, y, z) ;\left(\vec{w}_{\epsilon}^{3 *}\right)_{2}(x, y, z)=\left(\vec{w}_{\epsilon}^{1 *}\right)_{2}(z, y, x) \\
& \left(\vec{w}_{\epsilon}^{3 *}\right)_{3}(x, y, z)=\left(\vec{w}_{\epsilon}^{1 *}\right)_{1}(z, y, x) ; q_{\epsilon}^{3 *}(x, y, z)=q_{\epsilon}^{1 *}(z, y, x) .
\end{aligned}
$$

Suppose $N=2$ and let $\rho$ be the quantity $\left(x^{2}+y^{2}\right)^{1 / 2}$. There exist four positive quantities $a_{\epsilon}, b_{\epsilon}, c_{\epsilon}$ and $d_{\epsilon}$ such that

$$
\vec{w}_{\epsilon}^{1 *}(x, y)=\operatorname{curl}\left(h_{\epsilon}(\rho) y\right), q_{\epsilon}^{1 *}(x, y)=k_{\epsilon}(\rho) x \text { in } B(\epsilon / 4),
$$

where

$$
\begin{aligned}
& h_{\epsilon}(\rho)=a_{\epsilon} \rho^{2}+B_{\epsilon} \rho^{-2}+c_{3} \log \rho+d_{\epsilon} \\
& k_{\epsilon}(\rho)=8 a_{\epsilon}-2 c_{\epsilon} \rho^{-2}
\end{aligned}
$$




\section{A. Brillard}

Moreover, $a_{\epsilon}$ is equivalent to $\left(\epsilon^{2} \log \left(\epsilon / r_{\epsilon}\right)\right)^{-1}, b_{\epsilon}$ is equivalent to $r_{\epsilon}^{2} /\left(\log \left(\epsilon / r_{\epsilon}\right)\right), c_{\epsilon}$ is equivalent to $\left(\log \left(\epsilon / r_{\epsilon}\right)\right)^{-1}$ and $d_{\epsilon}$ is equivalent to $\epsilon\left(r_{\epsilon}\right)^{-3}$. The function $\vec{w}_{\epsilon}^{2 *}$ is defined by

$$
\begin{gathered}
\left(\vec{w}_{\epsilon}^{2 *}\right)_{1}(x, y)=\left(\vec{w}_{\epsilon}^{1 *}\right)_{2}(y, x) ;\left(\vec{w}_{\epsilon}^{2 *}\right)_{2}(x, y)=\left(\vec{w}_{\epsilon}^{1 *}\right)_{1}(y, x) ; \\
q_{\epsilon}^{2 *}(x, y)=q_{\epsilon}^{1 *}(y, x) .
\end{gathered}
$$

Proof of Proposition A.2. see [4]. - Let us come back to the proof of Proposition A.1.

b) is the consequence of the above computations through the following Lemma, extending a result of [5] (Lemma 2.3.) and proved in the same way.

LEMMA A.3.- Let $\delta_{\epsilon}^{i}$ be the Dirac mass uniformly distributed on $\partial B^{i}(\epsilon / 4)$. Then

$$
\sum_{i} \epsilon \delta_{\epsilon}^{i} \frac{s-W^{-1, \infty}(\Omega)}{\epsilon \rightarrow 0} \rightarrow \pi
$$

If $N=3$, denote $\rho_{\epsilon i}=\left(\left(x-x_{\epsilon i}\right)^{2}+\left(y-y_{\epsilon i}\right)^{2}+\left(z-z_{\epsilon i}\right)^{2}\right)^{1 / 2}$, then the three sequences

$$
\left(\sum_{i} \epsilon \frac{\left(x-x_{\epsilon i}\right)^{2}}{\rho_{\epsilon i}^{2}} \delta_{\epsilon}^{i}\right)_{\epsilon},\left(\sum_{i} \epsilon \frac{\left(y-y_{\epsilon i}\right)^{2}}{\rho_{\epsilon i}^{2}} \delta_{\epsilon}^{i}\right)_{\epsilon},\left(\sum_{i} \epsilon \frac{\left(z-z_{\epsilon i}\right)^{2}}{\rho_{\epsilon i}^{2}} \delta_{\epsilon}^{i}\right)_{\epsilon}
$$

converge in the strong topology of $W^{-1, \infty}(\Omega)$ to $\pi / 3$. The three sequences

$$
\begin{gathered}
\left(\sum_{i} \epsilon \frac{\left(x-x_{\epsilon i}\right)\left(y-y_{\epsilon i}\right)}{\rho_{\epsilon i}^{2}} \delta_{\epsilon}^{i}\right)_{\epsilon},\left(\sum_{i} \epsilon \frac{\left(x-x_{\epsilon i}\right)\left(z-z_{\epsilon i}\right)}{\rho_{\epsilon i}^{2}} \delta_{\epsilon}^{i}\right)_{\epsilon}, \\
\left(\sum_{i} \epsilon \frac{\left(y-y_{\epsilon i}\right)\left(z-z_{\epsilon i}\right)}{\rho_{\epsilon i}^{2}} \delta_{\epsilon}^{i}\right)_{\epsilon}
\end{gathered}
$$

converge to 0 in the strong topology of $W^{-1, \infty}(\Omega)$.

If $N=2$, denote $\rho_{\epsilon i}=\left(\left(x-x_{\epsilon i}\right)^{2}+\left(y-y_{\epsilon i}\right)^{2}\right)^{1 / 2}$, then the two sequences

$$
\left(\sum_{i} \epsilon \frac{\left(x-x_{\epsilon i}\right)^{2}}{\rho_{\epsilon i}^{2}} \delta_{\epsilon}^{i}\right)_{\epsilon} \text { and }\left(\sum_{i} \epsilon \frac{\left(y-y_{\epsilon i}\right)^{2}}{\rho_{\epsilon i}} \delta_{\epsilon}^{i}\right)_{\epsilon}
$$

converge in the strong topology of $W^{-1, \infty}(\Omega)$ to $\pi / 2$. The sequence

$$
\begin{gathered}
\left(\sum_{i} \epsilon \frac{\left(x-x_{\epsilon i}\right)\left(y-y_{\epsilon i}\right)}{\rho_{\epsilon i}^{2}} \delta_{\epsilon}^{i}\right)_{\epsilon} \\
-250-
\end{gathered}
$$


converges to 0 in the strong topology of $W^{-1, \infty}(\Omega)$.

Finally, we mention the following result concerning the pressure $q_{\epsilon}^{k}$ in (4). This result is useful in the study of the behaviour of the internal pressure of the fluid (Theorem 3.2).

LEMMA A.4. - Suppose $T$ is equal to $B(1)$ and $\alpha_{N}$ is finite. Then the sequence $\left(\chi_{\cup_{i} B^{i}(\epsilon / 4) \backslash T_{\epsilon i}} q_{\epsilon}^{k}\right)_{\epsilon}$, which is bounded in $L^{2}(\Omega)$, converges in the weak topology of this space to a constant.

Proof of Lemma A.4. - Take $\vec{v}$ in $\left(C_{o}^{\infty}(\Omega)\right)^{N}$ and compute, using (4)

$$
\begin{aligned}
\int_{\Omega_{\epsilon}} \operatorname{grad} \vec{w}_{\epsilon}^{k} \cdot \operatorname{grad}\left(\sum_{\ell} \vec{w}_{\epsilon}^{\ell} v_{\ell}\right) d x= & \sum_{i} \int_{B^{i}(\epsilon / 4)} q_{\epsilon}^{k}\left(\sum_{\ell} \vec{w}_{\epsilon}^{\ell} \cdot \operatorname{grad} v_{\ell}\right) d x+ \\
& +\sum_{i} \int_{\partial B^{i}(\epsilon / 4)}\left(\frac{\partial \vec{w}_{\epsilon}^{k}}{\partial \nu}-q_{\epsilon}^{k} \vec{\nu}\right) \vec{v} d \sigma_{\epsilon}(x) .
\end{aligned}
$$

Then

$$
\begin{aligned}
& \sum_{\ell} \int_{\Omega} \operatorname{grad} \vec{w}_{\epsilon}^{k} \cdot \operatorname{grad} \vec{w}_{\epsilon}^{\ell} v_{\ell} d x+\sum_{\ell} \int_{\Omega} \operatorname{grad} \vec{w}_{\epsilon}^{k} \operatorname{grad} v_{\ell} \vec{w}_{\epsilon}^{\ell} d x= \\
& =\sum_{i} \int_{B^{i}(\epsilon / 4)} q_{\epsilon}^{k}\left(\sum_{\ell} \vec{w}_{\epsilon}^{\ell} \cdot \operatorname{grad} v_{\ell}\right) d x \\
& +\sum_{i} \int_{\partial_{B^{i}(\epsilon / 4)}}\left(\frac{\partial \vec{w}_{\epsilon}^{k}}{\partial \nu}-q_{\epsilon}^{k} \vec{\nu}\right) \vec{v} d \sigma_{\epsilon}(x) .
\end{aligned}
$$

From the regularity of $\vec{v}$ and the definition of the matrix $M(3)$, one derives, using Proposition A.1.

$$
\sum_{\ell} \int_{\Omega} M_{k \ell} v_{\ell} d x=\int_{\Omega} q^{k} \operatorname{div} \vec{v} d x+\sum_{\ell} \int_{\Omega} M_{k \ell} v_{\ell} d x,
$$

where $q^{k}$ is a limit point of the sequence $\left(\chi_{\cup i B^{i}(\epsilon / 4) \backslash T_{\epsilon i}} q_{\epsilon}^{k}\right)_{\epsilon}$, in the weak topology of $L^{2}(\Omega)$ (the existence of $q^{k}$ is a consequence of the computations given in Proposition A.2). 


\section{A. Brillard}

\section{Références}

[1] Adams (R.A.). - Sobolev Spaces. - Academic Press, 1975.

[2] Atrouch (H.). - Variational convergence for functions and operators. Applicable Mathematics series. - Pitman (London), 1984.

[3] Atrouch (H.), and Picard (C.). - Variational inequalities with varying obstacles; the general form of the limit problem, J.Functional Analysis, t. 50 (3), 1983, p. 329-386.

[4] BRILLARD (A.). - Ecoulement d'un fluide incompressible dans un milieu poreux. Publication Avamac (Perpignan), 1985.

[5] Cioranescu (D.), and Murat (F.). - Un terme étrange venu d'ailleurs, Collège de France Seminar. Research Notes in Maths, t. 60, 70 Pitman (London), 1982.

[6] De Grorgi (E.). - Convergence problems for functionals and operators. Proc. Int. Congress on "Recent Methods in Nonlinear Analysis" Rome 1978 De Giorgi, Magenes, Mosco Eds. Pitagora Editrice (Bologna) 1979.

[7] LADYZHENSKAYA (O.A.). - The mathematical theory of viscous incompressible flow. - Gordon and Breach (New York), 1963.

[8] Levy (T.).- Loi de Darcy ou loi de Brinkman?, C.R.A.S. série II, t. 292, 1981, p. 871-874.

[9] Levy (T.). - Fluid flow through an array of fixed particles, Int. J. Engin. Sci., t. 21 $\mathrm{n}^{\circ} 1,1983$, p. $11-23$.

[10] Marchenko, Hrouslov .- Problèmes aux limites dans des domaines aux frontières finement granulées. - in russian. Naukova Dumka (Kiev), 1974 .

[11] Murat (F.). - Oral communication-1985.

[12] SANCHEZ-PALENCIA (E.). - Non-homogeneous media and vibration theory. - Lectures Notes in Physics $n^{\circ} 127$. Springer-Verlag (Berlin), 1980.

[13] TA RTAR (L.). - Incompressible fluid flow in a porous medium. Convergence of the homogenization process. - Appendix in the preceding reference [12].

[14] Temam (R.). - Navier-Stokes equations. - North Holland (Amsterdam), 1977.

Brinkman's law may be found in the original paper.

BrinkMAN (H.C.). - A calculation of the viscous force exerted by a flowing fluid on a dense swarm of particles, Appli. Sci. Res., t. A1, 1947, p. 27-34.

(Manuscrit reçu le 26 mai 1986) 